\title{
Closed Form Probabilistic Analysis of Lamination Parameters for Composite Structures
}

\author{
Benedikt Kriegesmann \\ Hamburg University of Technology, Am Schwarzenberg-Campus 4, 21073 Hamburg, Germany
}

Probabilistic analyses allow predicting the stochastic distribution of an output variable (e.g., the buckling load of a structure) based on the stochastic distribution of input parameters (e.g., material and geometric properties). In the probabilistic analysis of composite structures, one important quantity that can be subject to scatter is the ply or fiber orientation of the layers. Laminates with a large number of plies lead to a large number of random variables, which makes the probabilistic analysis very time consuming. Lamination parameters allow describing any ply layup by a maximum of 12 parameters. They have therefore been utilized for the design optimization of thick laminates. In the current paper, a two-step procedure is considered for utilizing lamination parameters for probabilistic analyses of composite structures with many plies. In the first step, the stochastic distribution of lamination parameters is determined. In the second step, the actual probabilistic analysis is performed. A closed form solution for the stochastic moment of lamination parameters is presented. Furthermore, the paper discusses under which circumstances lamination parameters are approximately normally distributed, which allows increasing the efficiency of both steps.

\section{Nomenclature}

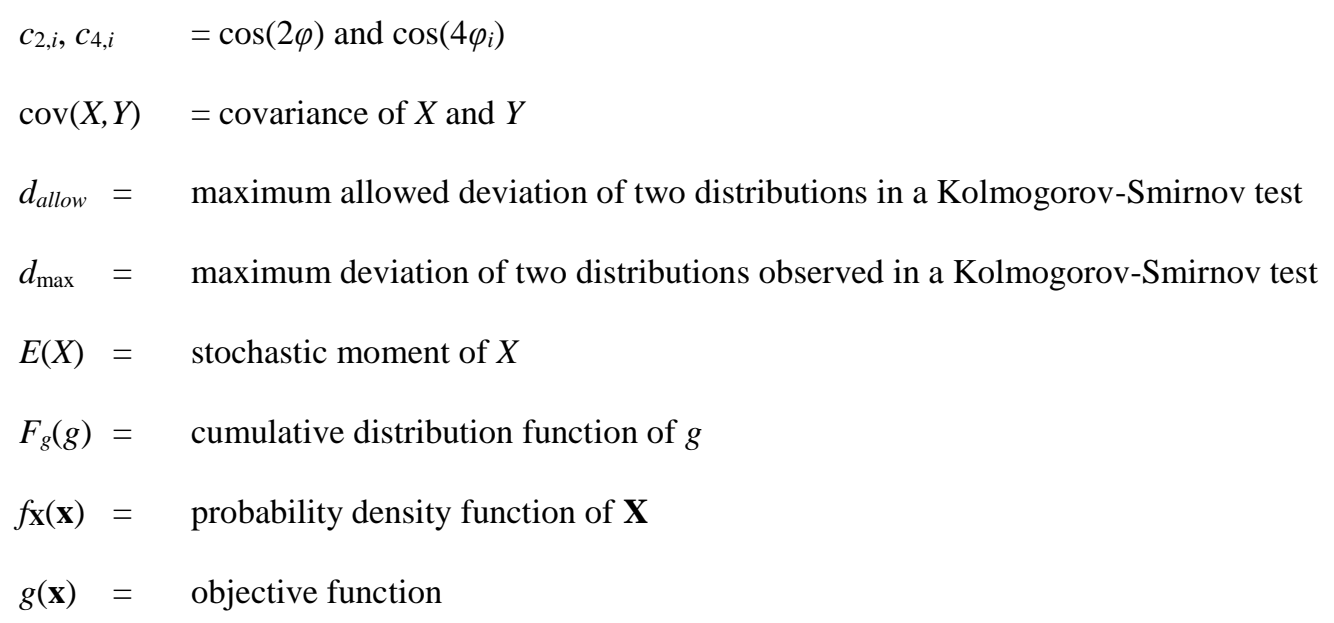




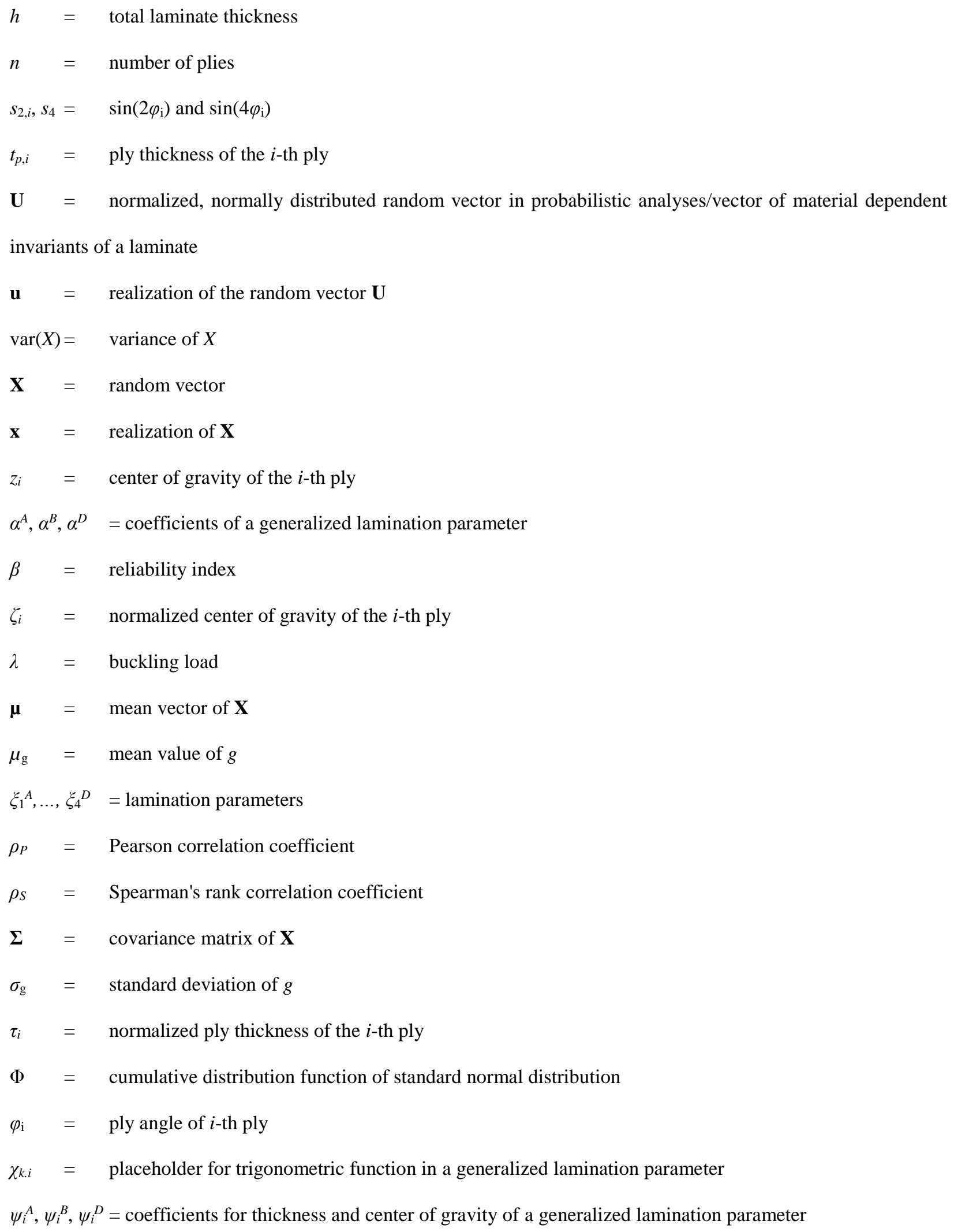




\section{I.Introduction}

THE response of structures in general is subject to scatter due to the randomness of parameters such as material properties, geometric measures or boundary conditions. In engineering practices, all sources of scatter are captured by applying knockdown and/or safety factors. The accumulation of these factors leads to an overly conservative design, which can be overcome by probabilistic sizing approaches [1]. Probabilistic analyses allow the prediction of the structural response due to the scatter of parameters. Given the stochastic distribution of the structural response (e.g., the failure load), the allowable applied load can be defined by choosing a probability of failure. (see, e.g., [2,3]).

A parameter specific to composite structures is the ply orientation, which is also random in nature. Only few works consider the scatter of ply orientations in probabilistic analyses, though it can have a significant impact on the scatter of the structural response (see, e.g., [4,5]). The reason is that there is typically no information of the stochastic distribution of this measure.

When considering ply orientations as random parameters in probabilistic analyses, the number of parameters increases with the number of plies. In Monte Carlo simulations, a large number of samples is generated and tested virtually. The computational effort does not depend on the number of random parameters, but it is typically very large compared to other approaches. In contrast to that, for computationally less expensive approaches the effort typically increases with the number of random parameters. Approaches like the first-order second-moment (FOSM) method and first order reliability method (FORM), which are introduced later in detail, the derivatives of the objective function have to be known. Since these are often estimated by finite differences (see, e.g. [6,7]), the numerical effort increases with the number of random parameters. Hence, when analyzing a composite structure with many layers, taking into account the ply orientation increases the computational cost significantly.

Tsai and Hahn [8] introduced the concept of lamination parameters, which offers an efficient way to describe a layup with a small number of parameters. (In [8] the lamination parameters are referred to as "geometric factors".) Therefore, lamination parameters have been suggested to be used for optimization of composite structures by Miki [9] and Fukunaga and Hirano [10]. Miki and Sugiyama [11] and Fukunaga and Sekine [12] also provided the feasible regions of lamination parameters, which are essential for optimization purposes. While these publications consider plates or simple shell structures, lamination parameters have been demonstrated to be useful for optimizing even complex structures like variable stiffness composites [13] or stiffened aircraft fuselage structures [14]. 
A similar way to express the laminate stiffness by a reduced number of variables is the Polar method, which has also been utilized for design optimization [15-17]. This work, however, focuses on the concept of lamination parameters and their utilization for probabilistic analyses.

Lamination parameters were first used for reliability-based design optimization by Miki et al. [18], where a probabilistic analysis was performed as part of the optimization constraints. In a similar way, Kogiso et al. [19] presented a reliability-based design optimization with lamination parameters in which material properties are considered as random. The approach has been extended to also consider the load as random in [20]. The abovementioned works focused on the reliability-based design optimization strategy, and therefore either lamination parameters were not considered as random, or no information was given on how the distribution of ply orientations was related to the distribution of lamination parameters.

Scarth et al. [21] performed a probabilistic analysis of the aeroelastic stability of composite plate wings where the lamination parameters themselves were considered as random parameters. The stochastic distribution of lamination parameters was determined from a Monte Carlo simulation. This distribution was then considered as an input for the actual probabilistic analysis, which was performed by a polynomial chaos expansion. This strategy of performing a probabilistic analysis in two steps is followed in the current paper. In the first step, the distribution of lamination parameters is determined and in the second step, the actual probabilistic analysis of the structural response is performed, considering the lamination parameters as random input parameters. Performing a probabilistic analysis in two steps can actually decrease the computational cost, as discussed in this paper. Different possibilities for utilizing lamination parameters for probabilistic analyses are discussed in section II of the current paper. The current work, however, focuses on the first step. A closed form solution of the mean values, variances and covariances of lamination parameters is presented in section III. Furthermore, it is discussed in section IV under which circumstances the distribution of lamination parameters can be assumed to be Gaussian and why this is beneficial.

\section{II.Utilizing lamination parameters for probabilistic analysis}

When performing a probabilistic analysis of a composite structure in which the angles $\varphi_{i}$ of each ply are considered as random parameters, the number of random parameters equals the number of plies $n$. For the Taylor series-based probabilistic approaches discussed in sections II.B and I.C, the computational effort for running the probabilistic analysis increases with the number of random parameters. 


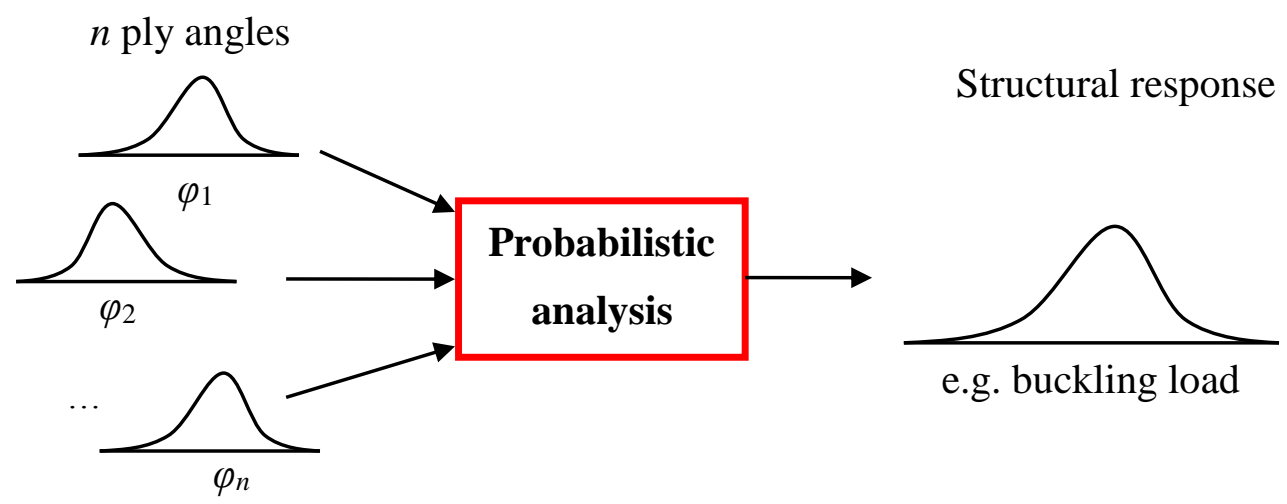

Fig. 1 Direct probabilistic analysis with ply angles as random parameters.

Lamination parameters allow the decoupling of the stiffness matrix of a laminate (ABD matrix) into a set of parameters purely dependent on material properties (invariants $\mathbf{U}$ ), another set purely dependent on the layer orientations (lamination parameters) and the laminate thickness. All relevant equations and definitions are summarized in Appendix A.

The fact that lamination parameters allow us to describe a layup with 12 parameters can be utilized to reduce the number of random parameters in a probabilistic analysis. Instead of determining the scatter in the structural response directly from the scatter of ply angles as shown in Fig. 1, an intermediate step is required to first determine the distribution of lamination parameters, as shown in Fig. 2. For the probabilistic analysis of the lamination parameters (PA1 in Fig. 2), the objective function is given explicitly, while in the second step (PA2 in Fig. 2), the objective function can, for instance, be the result of a finite element analysis. Therefore, the first analysis is performed much more quickly than the second step, and it is therefore worth running it in order to reduce the number of parameters in the second step. 


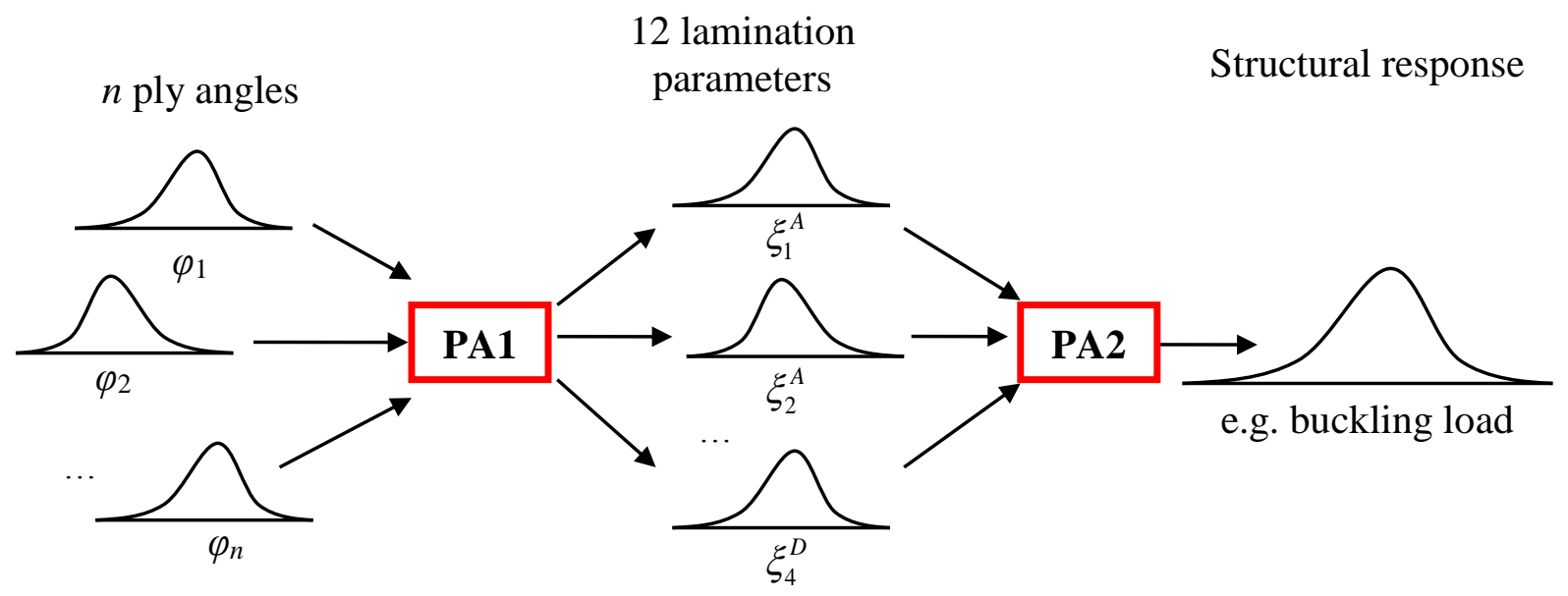

Fig. 2 Indirect probabilistic analysis with ply angles as random parameters, using lamination parameters.

This procedure only makes sense if the number of plies is larger than 12 , since in general the number of lamination parameters equals 12. Under certain assumptions, the number of lamination parameters can be reduced. Symmetric laminates are described by only eight parameters. When considering only $0^{\circ}, \pm 45^{\circ}$ and $90^{\circ}$ ply angles, the number of parameters can be further reduced to six (see, e.g., $[11,14])$. These assumptions can be useful for optimization tasks. However, within a probabilistic analysis, a symmetrical laminate can become unsymmetrical due to the scatter of the ply angles. Therefore, all 12 lamination parameters need to be considered.

In the following sections, different probabilistic approaches are described that allow performance of a probabilistic analysis, as illustrated in Fig. 1 and each of the steps shown in Fig. 2. The probabilistic methods themselves are not the subject of the current paper, and are therefore discussed very briefly. For comprehensive descriptions of the methods, refer to [2]. As a general notation, consider the objective function $g(\mathbf{x})$, which is a function of realizations $\mathbf{x}$ of the random vector $\mathbf{X}$ with the probability density function $f \mathbf{x}(\mathbf{x})$. The objective of the probabilistic approaches is to determine the stochastic distribution $F_{g}(g)$ of the objective function $g$ due to the scatter of $\mathbf{X}$.

\section{A. Monte Carlo method}

For the Monte Carlo method, a large number of realizations $\mathbf{x}^{(i)}$ of the random vector $\mathbf{X}$ is generated according to its distribution $f_{\mathbf{x}}(\mathbf{x})$. For each realization, the objective function is evaluated $g\left(\mathbf{x}^{(i)}\right)=g^{i}$ and thereby a discrete distribution of $g$ is obtained. The number of realizations that need to be evaluated is determined by a convergence 
check during the simulation. The Monte Carlo method is typically the computationally most expensive method, but it also provides a very robust and easy to use algorithm.

Several techniques exist which improve the efficiency of Monte Carlo simulations, such as importance sampling or response surface methods (see, e.g., [2]).

The computational cost of a Monte Carlo simulation does not necessarily increase with the number of random parameters. Hence, if the probabilistic analysis of the structural response is performed with Monte Carlo techniques it may not beneficial to use the two-step approach shown in Fig. 2.

\section{B. Approximation at the mean vector - FOSM}

The first-order second-moment (FOSM) method utilizes a Taylor series expansion of the objective function at the mean vector $\boldsymbol{\mu}$ of $\mathbf{X}$. Inserting this into the definition of the mean value $\mu_{g}$ of $g$ yields the following approximation, when only linear terms of the Taylor series are considered.

$$
\mu_{g} \approx g(\boldsymbol{\mu})
$$

In a similar manner, the variance $\sigma_{g}{ }^{2}$ of $g$ is approximated.

$$
\sigma_{g}^{2} \approx \sum_{i=1}^{n} \sum_{j=1}^{n} \frac{\partial g(\boldsymbol{\mu})}{\partial x_{i}} \frac{\partial g(\boldsymbol{\mu})}{\partial x_{j}} \operatorname{cov}\left(X_{i}, X_{j}\right)
$$

where $n$ is the length of $\mathbf{X}, \partial g(\boldsymbol{\mu}) / \partial x_{i}$ is the partial derivative at $\boldsymbol{\mu}$ and $\operatorname{cov}\left(X_{i}, X_{j}\right)$ is the covariance of the $i$-th and $j$-th entry of $\mathbf{X}$.

If the objective function is not given explicitly, the partial derivatives must be approximated, e.g., by central differences. Thereby, $2 n+1$ evaluations of the objective function are required, which is typically a significantly smaller number of evaluations compared to the Monte Carlo simulation.

For the FOSM approach, it is not necessary to know the distribution function of $\mathbf{X}$; only the stochastic moments are required. FOSM does not provide the distribution function of $g$, but only its mean and variance. A distribution function $F_{g}(g)$ can be obtained if a reasonable assumption for the type of distribution (e.g., Gauss) is possible.

The FOSM method is a good candidate for the second step of the two-step approach shown in Fig. 2, as it will always only require $2 \cdot 12+1=25$ evaluations of the structural response.

\section{Approximation at the most probable point - FORM}


While a Monte Carlo simulation provides the whole cumulative distribution function $F_{g}(g)$, the approach summarized in the following only intends to determine the cumulative frequency $F_{g}\left(g^{*}\right)$ of a certain value $g^{*}$. The cumulative frequency $F_{g}\left(g^{*}\right)$ gives the probability that $g(\mathbf{x})$ does not exceed $g^{*}$. This is achieved by a Taylor series approximation of $g(\mathbf{x})$ at the so-called most probable point (MPP). The MPP is the point on $g(\mathbf{x})=g^{*}$ with the highest probability density $f_{\mathbf{x}}(\mathbf{x})$ (see Fig. 3).

When transforming $\mathbf{X}$ to a vector of uncorrelated, standard normally distributed variables $\mathbf{U}$, the MPP is the point on $g(\mathbf{u})=g^{*}$ with the smallest distance to the mean/origin, which is called the reliability index $\beta$. When $\beta$ is determined, the probability $P\left(g(\mathbf{x}) \leq g^{*}\right)=\Phi(-\beta)$ can be determined, where $\Phi$ is the standard normal distribution. If $g$ is not linear or becomes nonlinear by the transformation from $\mathbf{X}$ to $\mathbf{U}$, this approach is no longer exact, but is an approximation; hence $P\left(g(\mathbf{x}) \leq g^{*}\right) \approx \Phi(-\beta)$.
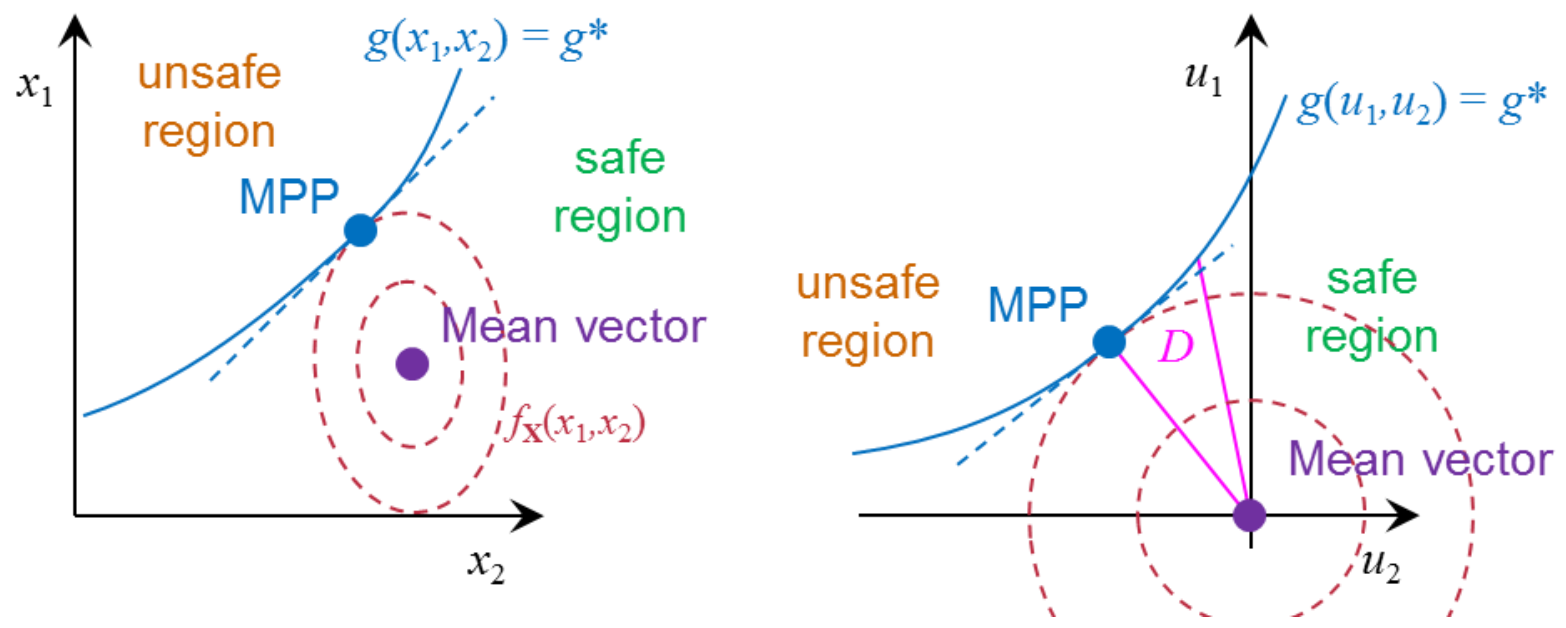

Fig. 3 Most probable point (MPP) in original and normalized space.

If $\mathbf{X}$ is already normally distributed and $g$ is linear, the MPP, and thereby $\beta$, can be determined directly. Otherwise, the MPP needs to be found by solving the optimization problem:

$$
\begin{aligned}
& \text { minimize } D \text {, with } D=\sqrt{\mathbf{u}^{T} \mathbf{u}} \\
& \text { subject to } g(\mathbf{u})=g *
\end{aligned}
$$

If the random vector $\mathbf{X}$ is normally distributed, the transformation to $\mathbf{U}$ is given by the following linear transformation. 


$$
\mathbf{x}=\boldsymbol{\Sigma}^{\frac{1}{2}} \mathbf{u}+\boldsymbol{\mu}
$$

Here, $\boldsymbol{\Sigma}$ is the covariance matrix and $\boldsymbol{\mu}$ is the mean vector of $\mathbf{X}$. If, however, the $\mathbf{X}$ follows any other distribution, the Rosenblatt transformation (see, e.g., [2]) needs to be utilized to transform $\mathbf{X}$ to $\mathbf{U}$. In the latter case, an objective function that is linear in $\mathbf{x}$ can become nonlinear in $\mathbf{u}$.

The FORM method can be used for the second step of the two-step approach shown in Fig. 2. For determining the gradients in the optimization loops $2 \cdot 12+1=25$, evaluations of the structural response are required.

\section{III.Stochastic moments of lamination parameters}

In the following, a direct determination of mean values, variances and covariances of lamination parameters is presented.

\section{A. Preliminary remarks}

Consider a laminate with $n$ plies with ply thicknesses $t_{p, 1}, \ldots, t_{p, n}$, the total thickness $h$, the ply orientations $\varphi_{1}, \ldots, \varphi_{n}$ and the centers of gravity $z_{1}, \ldots, z_{n}$ of each ply. Introducing

$$
c_{2, i}=\cos \left(2 \varphi_{i}\right) \quad, \quad c_{4, i}=\cos \left(4 \varphi_{i}\right) \quad, \quad s_{2, i}=\sin \left(2 \varphi_{i}\right) \quad \text { and } \quad s_{4, i}=\sin \left(4 \varphi_{i}\right)
$$

and

$$
\tau_{i}=\frac{t_{p, i}}{h} \quad \zeta_{i}=\frac{z_{i}}{h}
$$

the lamination parameters can be written as

$$
\begin{aligned}
& \xi_{[1,2,3,4]}^{A}=\sum_{i=1}^{n}\left[c_{2, i}, c_{4, i}, s_{2, i}, s_{4, i}\right] \tau_{i} \\
& \xi_{[1,2,3,4]}^{B}=4 \sum_{i=1}^{n}\left[c_{2, i}, c_{4, i}, s_{2, i}, s_{4, i}\right] \zeta_{i} \tau_{i} \\
& \xi_{[1,2,3,4]}^{D}=12 \sum_{i=1}^{n}\left[c_{2, i}, c_{4, i}, s_{2, i}, s_{4, i}\right]\left(\zeta_{i}^{2} \tau_{i}+\frac{\tau_{i}^{3}}{12}\right)
\end{aligned}
$$

It is assumed that any scatter in laminate thickness $h$ is distributed equally among all plies. Thus, the normalized values $\tau_{i}$ and $\zeta_{i}$ are not affected by the variability of $h$ and are therefore not random parameters.

\section{B. Mean values of lamination parameters}

Exemplarily consider the lamination parameter $\xi_{1}{ }^{A}$, for which the mean value is given by 


$$
E\left(\xi_{1}^{A}\right)=E\left(\sum_{i=1}^{n} c_{2, i} \tau_{i}\right)=\sum_{i=1}^{n} E\left(c_{2, i}\right) \tau_{i}
$$

Hence, the mean values of $c_{2, i}$ have to be determined, which are given by

$$
E\left(c_{2, i}\right)=\int_{-\infty}^{\infty} \cos \left(2 \varphi_{i}\right) f_{\Phi}\left(\varphi_{i}\right) d \varphi_{i}
$$

The solution of this integral obviously depends on the type of distribution of $\varphi_{i}$. If $\varphi_{i}$ is uniformly distributed, the integral can be solved exactly as is given in Appendix D. A more general solution of this integral is given in Appendix B by making use of the series expansion of cosine. Since for this series an upper bound needs to be chosen in practice, the solution is only approximate. Furthermore, it requires knowledge about some very high-order moments. For normal distribution, a general formulation of these high order moments is available and given at the end of Appendix B.

In the same manner, the mean values of all lamination parameters are derived and given by

$$
\begin{aligned}
& E\left(\xi_{[1,2,3,4]}^{A}\right)=\sum_{i=1}^{n}\left[E\left(c_{2, i}\right), E\left(c_{4, i}\right), E\left(s_{2, i}\right), E\left(s_{4, i}\right)\right] \tau_{i} \\
& E\left(\xi_{[1,2,3,4]}^{B}\right)=4 \sum_{i=1}^{n}\left[E\left(c_{2, i}\right), E\left(c_{4, i}\right), E\left(s_{2, i}\right), E\left(s_{4, i}\right)\right] \zeta_{i} \tau_{i} \\
& E\left(\xi_{[1,2,3,4]}^{D}\right)=12 \sum_{i=1}^{n}\left[E\left(c_{2, i}\right), E\left(c_{4, i}\right), E\left(s_{2, i}\right), E\left(s_{4, i}\right)\right]\left(\zeta_{i}^{2} \tau_{i}+\frac{\tau_{i}^{3}}{12}\right)
\end{aligned}
$$

The mean values of $c_{2, i}, c_{4, i}, s_{2, i}$ and $s_{4, i}$ are given in Appendix D for uniformly distributed $\varphi_{i}$ and in Appendix B for any distribution, such as normal distribution.

\section{Variances of lamination parameters}

Exemplarily consider $\xi_{1}^{A}$, for which the variance is given by

$$
\operatorname{var}\left(\xi_{1}^{A}\right)=\operatorname{var}\left(\sum_{i=1}^{n} c_{2, i} \tau_{i}\right)
$$

It is assumed that the scatter of a ply angle is independent of the scatter of any other ply angle; hence, each $\varphi_{i}$ is uncorrelated with any other $\varphi_{j}(i \neq j)$. Then, $c_{2, i}$ and $c_{2, j}$ are also uncorrelated, which allows the Bienaymé formula for the variance of a sum of uncorrelated variables to be used [22]. 


$$
\operatorname{var}\left(\sum_{i=1}^{n} c_{2, i} \tau_{i}\right)=\sum_{i=1}^{n} \operatorname{var}\left(c_{2, i} \tau_{i}\right)
$$

Exploiting the assumption that any scatter in the laminate thickness is distributed equally among all plies allows removing the normalized ply thickness from the variance calculation.

$$
\sum_{i=1}^{n} \operatorname{var}\left(c_{2, i} \tau_{i}\right)=\sum_{i=1}^{n} \operatorname{var}\left(c_{2, i}\right) \tau_{i}^{2}
$$

Now, only the variances of $c_{2, i}$ need to be determined.

$$
\operatorname{var}\left(c_{2, i}\right)=\int_{-\infty}^{\infty} \cos ^{2}\left(2 \varphi_{i}\right) f_{\Phi_{i}}\left(\varphi_{i}\right) d \varphi_{i}-E^{2}\left(c_{2, i}\right)
$$

Using the power reduction formula (a.k.a., the substitution of variables) for the cosine

$$
\cos ^{2}(x)=\frac{1+\cos (2 x)}{2}
$$

yields

$$
\begin{aligned}
\operatorname{var}\left(c_{2, i}\right) & =\int_{-\infty}^{\infty} \frac{1+\cos \left(4 \varphi_{i}\right)}{2} f_{\Phi_{i}}\left(\varphi_{i}\right) d \varphi_{i}-E^{2}\left(c_{2, i}\right) \\
& =\frac{1}{2} \underbrace{\int_{-\infty}^{\infty} f_{\Phi_{i}}\left(\varphi_{i}\right) d \varphi_{i}}_{=1}+\underbrace{\frac{1}{2} \int_{-\infty}^{\infty} \cos \left(4 \varphi_{i}\right) f_{\Phi_{i}}\left(\varphi_{i}\right) d \varphi_{i}}_{E\left(c_{4, i}\right)}-E^{2}\left(c_{2, i}\right) \\
& =\frac{1}{2}+\frac{1}{2} E\left(c_{4, i}\right)-E^{2}\left(c_{2, i}\right)
\end{aligned}
$$

Again, the mean values of the trigonometric functions are required, which are given in Appendix B and Appendix D, respectively.

Inserting (16) into (12) yields

$$
\operatorname{var}\left(\xi_{1}^{A}\right)=\sum_{i=1}^{n}\left[\frac{1}{2}+\frac{1}{2} E\left(c_{4, i}\right)-E^{2}\left(c_{2, i}\right)\right] \tau_{i}^{2}
$$

In the same manner, the variances of all other lamination parameters are derived. In general, they are given by 


$$
\begin{aligned}
& \operatorname{var}\left(\xi_{[1,2,3,4]}^{A}\right)=\sum_{i=1}^{n}\left[\operatorname{var}\left(c_{2, i}\right), \operatorname{var}\left(c_{4, i}\right), \operatorname{var}\left(s_{2, i}\right), \operatorname{var}\left(s_{4, i}\right)\right] \tau_{i}^{2} \\
& \operatorname{var}\left(\xi_{[1,2,3,4]}^{B}\right)=16 \sum_{i=1}^{n}\left[\operatorname{var}\left(c_{2, i}\right), \operatorname{var}\left(c_{4, i}\right), \operatorname{var}\left(s_{2, i}\right), \operatorname{var}\left(s_{4, i}\right)\right] \zeta_{i}^{2} \tau_{i}^{2} \\
& \operatorname{var}\left(\xi_{[1,2,3,4]}^{D}\right)=144 \sum_{i=1}^{n}\left[\operatorname{var}\left(c_{2, i}\right), \operatorname{var}\left(c_{4, i}\right), \operatorname{var}\left(s_{2, i}\right), \operatorname{var}\left(s_{4, i}\right)\right]\left(\zeta_{i}^{2} \tau_{i}+\frac{\tau_{i}^{3}}{12}\right)^{2}
\end{aligned}
$$

where the variances of $c_{2, i}, c_{4, i}, s_{2, i}$ and $s_{4, i}$ are given by

$$
\begin{aligned}
& \operatorname{var}\left(c_{2, i}\right)=\frac{1}{2}+\frac{1}{2} E\left(c_{4, i}\right)-E^{2}\left(c_{2, i}\right) \\
& \operatorname{var}\left(c_{4, i}\right)=\frac{1}{2}+\frac{1}{2} E\left(c_{8 i}\right)-E^{2}\left(c_{4, i}\right) \\
& \operatorname{var}\left(s_{2, i}\right)=\frac{1}{2}-\frac{1}{2} E\left(c_{4, i}\right)-E^{2}\left(s_{2, i}\right) \\
& \operatorname{var}\left(s_{4, i}\right)=\frac{1}{2}-\frac{1}{2} E\left(c_{8, i}\right)-E^{2}\left(s_{4, i}\right)
\end{aligned}
$$

In addition to the abbreviations introduced in III.A, $c_{8, i}=\cos \left(8 \varphi_{i}\right)$ is introduced. The appearance of cosine and a negative sign in the equations for $\operatorname{var}\left(s_{2, i}\right)$ and $\operatorname{var}\left(s_{4, i}\right)$ are caused by the power reduction formula, which for sine is given by

$$
\sin ^{2}(x)=\frac{1-\cos (2 x)}{2}
$$

\section{Covariances of lamination parameters}

Some lamination parameters are strongly correlated. Hence, it is essential to take their correlation into account in a probabilistic analysis.

Exemplarily consider the covariance of two lamination parameters $\xi_{1}^{A}$ and $\xi_{2}^{A}$.

$$
\operatorname{cov}\left(\xi_{1}^{A}, \xi_{2}^{A}\right)=E\left(\xi_{1}^{A} \xi_{2}^{A}\right)-E\left(\xi_{1}^{A}\right) E\left(\xi_{2}^{A}\right)
$$

The joint moment is given by

$$
E\left(\xi_{1}^{A} \xi_{2}^{A}\right)=E\left[\left(\sum_{i=1}^{n} c_{2, i} \tau_{i}\right)\left(\sum_{j=1}^{n} c_{4, j} \tau_{j}\right)\right]=E\left[\sum_{i=1}^{n} \sum_{j=1}^{n} c_{2, i} \tau_{i} c_{4, j} \tau_{j}\right]=\sum_{i=1}^{n} \sum_{j=1}^{n} E\left(c_{2, i} c_{4, j}\right) \tau_{i} \tau_{j}
$$

Separating terms for which $i=j$ and $i \neq j$ yields

$$
E\left(\xi_{1}^{A} \xi_{2}^{A}\right)=2 \sum_{i=1}^{n} \sum_{j=i+1}^{n} E\left(c_{2, i} c_{4, j}\right) \tau_{i} \tau_{j}+\sum_{i=1}^{n} E\left(c_{2, i} c_{4, i}\right) \tau_{i}^{2}
$$


Since the distributions of two different ply angles $\varphi_{i}$ and $\varphi_{j}($ for $i \neq j)$ are independent, $c_{i}$ and $c_{j}$ are also distributed independently. Therefore, the joint moment equals the product of the individual moments $E\left(\mathrm{c}_{2, i} c_{4, j}\right)=E\left(\mathrm{c}_{2, i}\right) E\left(c_{4, j}\right)$.

$$
\begin{aligned}
E\left(\xi_{1}^{A} \xi_{2}^{A}\right) & =2 \sum_{i=1}^{n} \sum_{j=i+1}^{n} E\left(c_{2, i}\right) E\left(c_{4, j}\right) \tau_{i} \tau_{j}+\sum_{i=1}^{n} E\left(c_{2, i} c_{4, i}\right) \tau_{i}^{2} \\
& =2 \sum_{i=1}^{n} E\left(c_{2, i}\right) \tau_{i} \sum_{j=i+1}^{n} E\left(c_{4, j}\right) \tau_{j}+\sum_{i=1}^{n} E\left(c_{2, i} c_{4, i}\right) \tau_{i}^{2}
\end{aligned}
$$

Hence, the moment of the product of the trigonometric functions needs to be determined, which is given in Appendix C for arbitrary distributions and in Appendix D for the uniform distribution of $\varphi_{i}$.

In the same manner, the covariances of all 66 combinations of lamination parameters are determined. In order to obtain a condensed format, consider a more general formulation of the lamination parameter.

$$
\xi_{k}^{K}=\alpha^{K} \sum_{i=1}^{n} \chi_{k, i} \psi_{i}^{K}
$$

with $K=A, B, C$ and $k=1,2,3,4$, where

$$
\begin{aligned}
& K=A \Rightarrow \alpha^{A}=1, \psi_{i}^{A}=\tau_{i} \\
& K=B \Rightarrow \alpha^{B}=4, \psi_{i}^{B}=\zeta_{i} \tau_{i} \\
& K=D \Rightarrow \alpha^{D}=12, \psi_{i}^{D}=\zeta_{i}^{2} \tau_{i}+\frac{\tau_{i}^{3}}{12}
\end{aligned}
$$

and

$$
\begin{aligned}
& k=1 \Rightarrow \chi_{k, i}=c_{2, i}=\cos \left(2 \varphi_{i}\right) \\
& k=2 \Rightarrow \chi_{k, i}=c_{4, i}=\cos \left(4 \varphi_{i}\right)
\end{aligned}
$$

Determining the covariance requires the joint moment of these generalized lamination parameters.

$$
\operatorname{cov}\left(\xi_{k}^{K}, \xi_{l}^{L}\right)=E\left(\xi_{k}^{K} \xi_{l}^{L}\right)-E\left(\xi_{k}^{K}\right) E\left(\xi_{l}^{L}\right)
$$

The joint moment is given by 


$$
\begin{aligned}
E\left(\xi_{k}^{K} \xi_{l}^{L}\right) & =E\left[\left(\alpha^{K} \sum_{i=1}^{n} \chi_{k, i} \psi_{i}^{K}\right)\left(\alpha^{L} \sum_{j=1}^{n} \chi_{l, j} \psi_{j}^{L}\right)\right] \\
& =\alpha^{K} \alpha^{L} \sum_{i=1}^{n} \sum_{j=1}^{n} E\left(\chi_{k, i} \chi_{l, j}\right) \psi_{i}^{K} \psi_{j}^{L} \\
& =\alpha^{K} \alpha^{L} \sum_{i=1}^{n}\left[E\left(\chi_{k, i}\right) \psi_{i}^{K} \sum_{j=1, j \neq i}^{n} E\left(\chi_{l, j}\right) \psi_{j}^{L}\right]+\alpha^{K} \alpha^{L} \sum_{i=1}^{n} E\left(\chi_{k, i} \chi_{l, i}\right) \psi_{i}^{K} \psi_{i}^{L}
\end{aligned}
$$

Hence, no matter for which combination of lamination parameters the covariance needs to be determined, the joint moment of the trigonometric functions $E\left(\chi_{k, i} \chi_{l, i}\right)$ needs to be determined. This is done for all combinations of $c_{2, i}, c_{4, i}$, $s_{2, i}$ and $s_{4, i}$ in Appendix C and Appendix D.

\section{IV.Validity of assuming normal distribution}

In the following chapter, it is discussed under which circumstances a normal (or Gaussian) distribution is a valid assumption for the distribution of lamination parameters.

The transformation from ply angles to lamination parameters is given by trigonometric functions. Fig. 4 illustrates what the stochastic distribution of cosine looks like depending on the value of the ply angle. For an angle $\varphi$ that scatters around $90^{\circ}$, the transformation by cosine is almost linear and, therefore, the distribution of $\cos (\varphi)$ is approximately of the same type as the distribution of $\varphi$ (represented by blue lines in Fig. 4). However, if $\varphi$ scatters around $0^{\circ}, \cos (\varphi)$ is concentrated at a value of 1 , while this is also the upper bound of the distribution (red lines in Fig. 4). For $\sin (\varphi)$, the same conclusions are valid when exchanging $0^{\circ}$ and $90^{\circ}$. Hence, the assumption that the lamination parameters are normally distributed is obviously not valid. However, it is shown in section IV.B that for a sufficiently large number of plies, it is a reasonable approximation to assume normal distribution for the lamination parameters. 


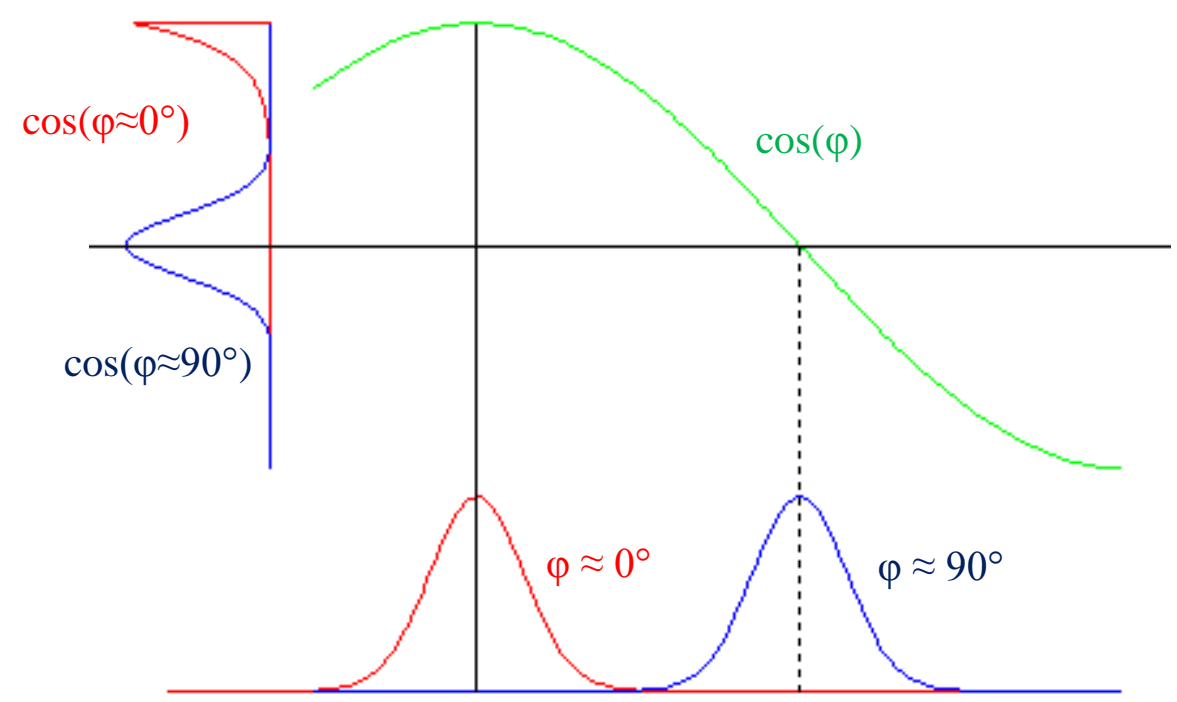

Fig. 4 Relation of the distributions of ply angle and cosine.

\section{A. Applicability of the central limit theorem}

Consider a sequence of independent and identically distributed random variables $X_{1}, X_{2}, \ldots, X_{n}$. The central limit theorem states that the sum $X$ of these samples converges towards normal distribution as $n \rightarrow \infty$ (see, e.g., [2,23]).

$$
X=\sum_{i=1}^{n} X_{i}
$$

The lamination parameters are given by a sum of random parameters. The summands can be assumed to be independent, but they are certainly not equally distributed in general. Consider exemplarily

$$
\xi_{1}^{D}=\sum_{i=1}^{n} \underbrace{12 \cos \left(2 \varphi_{i}\right)\left(\zeta_{i}^{2} \tau_{i}+\frac{\tau_{i}^{3}}{12}\right)}_{X_{i}}
$$

Here, the distribution of the summands depends on the distribution of $\varphi_{i}$. The parameters $\tau_{i}$ and $\zeta_{i}$ are not random, but only scale the summand. If the ply orientations $\varphi_{i}$ are independent of each other, the summands are independent. However, the distributions of the summands will differ as soon as the nominal values of the ply orientations differ, even if the distribution around the nominal value is the same. As shown, for instance, in Fig. 4, the same distribution around the nominal value of plies with $0^{\circ}$ and $90^{\circ}$ yields totally different distributions for $\cos \left(\varphi_{i}\right)$. Therefore, the 
central limit theorem is not applicable to lamination parameters, in general. (Note that in the lamination parameters equations the angles are multiplied by factor 2 or 4 , and therefore, the conclusion for $90^{\circ}$ holds for ply angles of $45^{\circ}$ or $22.5^{\circ}$.)

However, if the Linderberg condition holds, the distribution of $X$ converges towards normal distribution even if the parameters $X_{i}$ are not equally distributed (see, e.g., [23]). Linderberg's extension of the central limit theorem says that for random parameters $X_{i}$ with mean value $\mu_{i}$ and standard deviation $\sigma_{i}$, the sum

$$
Z=\frac{1}{s_{n}} \sum_{i=1}^{n}\left(X_{i}-\mu_{i}\right) \rightarrow N(0,1)
$$

converges towards standard normal distribution if

$$
\max _{k=1, \ldots, n} \frac{\sigma_{k}^{2}}{s_{n}^{2}} \rightarrow 0 \text { as } n \rightarrow \infty
$$

with

$$
s_{n}^{2}=\sum_{i=1}^{n} \sigma_{i}^{2}
$$

It is easy to show that if $Z$ converges towards standard normal distribution, $X$ converges toward normal distribution with mean value $m_{n}$ and standard deviation $s_{n}$.

$$
\begin{gathered}
Z=\frac{1}{s_{n}} \sum_{i=1}^{n}\left(X_{i}-\mu_{i}\right) \Leftrightarrow s_{n} Z=\sum_{i=1}^{n} X_{i}-\sum_{i=1}^{n} \mu_{i} \\
\Leftrightarrow s_{n} Z+\sum_{i=1}^{n} \mu_{i}=\sum_{i=1}^{n} X_{i}=X \rightarrow N\left(m_{n}, s_{n}^{2}\right) \\
m_{n}
\end{gathered}
$$

Hence, if the condition (33) holds for lamination parameters, the central limit theorem is also applicable to lamination parameters. The coefficient in (33) can be rewritten as

$$
\frac{\sigma_{k}^{2}}{s_{n}^{2}}=\frac{\sigma_{k}^{2}}{\sum_{i=1}^{n} \sigma_{i}^{2}}=\frac{\sigma_{k}^{2}}{\sigma_{k}^{2}+\sum_{\substack{i=1 \\ k \neq i}}^{n} \sigma_{i}^{2}}
$$

Practically, condition (33) requires that there is not one parameter $X_{k}$ with a standard deviation $\sigma_{k}$ that dominates the overall scatter. Proving that this condition is fulfilled for lamination parameters in general seems impossible to the author, because it is theoretically possible to consider a laminate that has one very thick ply with very large scatter in 
ply orientation $\left(\sigma_{k} \rightarrow \infty\right)$ and where all other plies are very small and have a very small scatter $\left(\sigma_{i} \rightarrow 0\right.$, for $\left.i \neq k\right)$. In practice, however, the plies usually have similar thicknesses and a similarly large scatter, even if the outer plies dominate due to $\zeta_{i}$ in (31). Then, the coefficient (36) will converge towards 0 as the number of plies $n$ increases and the central limit theorem is applicable.

\section{B. Convergence towards normal distribution in practical applications}

The following chapter tackles the question of whether lamination parameters are normally distributed from a more practical aspect. Even if the central limit theorem is valid, as discussed in the previous section, for practical applications it is essential to know for which number of plies normal distribution is a valid assumption. In Fig. 5, the cumulative distribution of lamination parameter $\xi_{2}{ }^{A}$ obtained from a Monte Carlo simulation is compared to a normal distribution with the same mean value and variance. If the layup consists of only one $0^{\circ}$ ply (Fig. 5 , left) the distribution obtained from Monte Carlo obviously differs significantly from a normal distribution. When considering a layup consisting of 13 independent $0^{\circ}$ plies, the distributions look very similar (see Fig. 5, right). In both cases, the ply orientations are assumed to be uniformly distributed in the interval $\pm 8.66^{\circ}$ of the nominal value, which yields a standard deviation of $5^{\circ}$ (compare [21]).
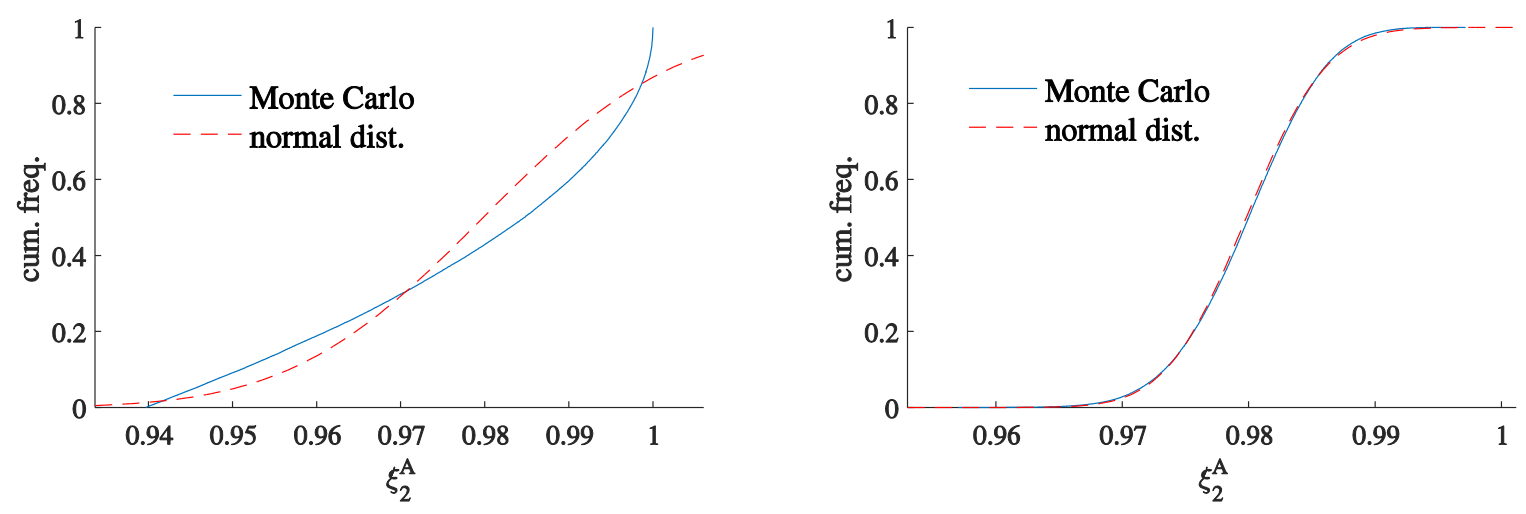

Fig. 5 Comparison of stochastic distributions determined by Monte Carlo simulation and direct solution for $\xi_{2}{ }^{A}$ of a single ply laminate with $\left[0^{\circ}\right]$ (left) and $\xi_{4}{ }^{A}$ of an 13 ply laminate with $\left[0^{\circ}\right]_{13}$ (right).

In order to quantify the convergence towards normal distribution, the Kolmogorov-Smirnov test (K-S test) is used

[2]. The cumulative distribution obtained from a Monte Carlo simulation $F_{M C}$ is given by 


$$
F_{M C}(x)=\frac{1}{m} \sum_{i=1}^{m} I_{x} \quad \text { with } I_{x}= \begin{cases}1 & \text { if } x_{i} \leq x \\ 0 & \text { if } x_{i}>x\end{cases}
$$

Here, $x_{i}$ are the realizations of the Monte Carlo simulation and $m$ is the number of realizations. For the K-S test, $F_{M C}$ is compared to the cumulative distribution function of a normal distribution $F_{N}$ with the same mean value and variance. The maximum deviation $d_{\max }$ of the two distributions is determined according to (38).

$$
\begin{array}{cc}
d_{U, i}=\left|F_{M C}\left(x_{i}\right)-F_{N}\left(x_{i}\right)\right| & d_{L, i}=\left|F_{M C}\left(x_{i-1}\right)-F_{N}\left(x_{i}\right)\right| \\
d_{i}=\max \left(d_{U, i}, d_{L, i}\right) & d_{\max }=\max \left(d_{1}, \ldots, d_{m}\right)
\end{array}
$$

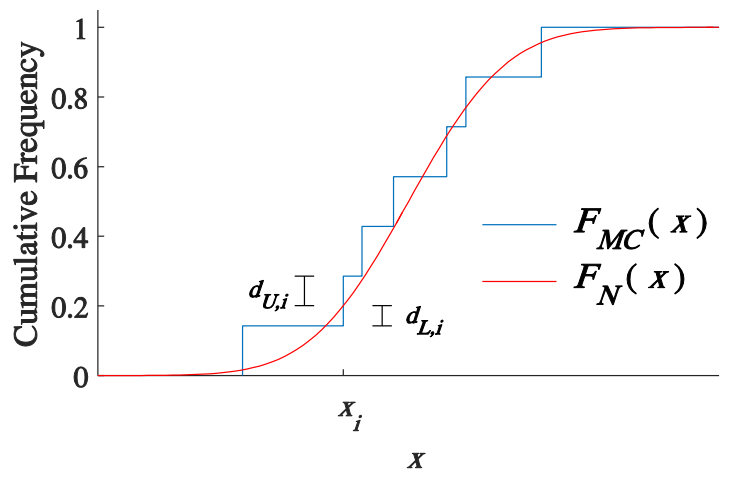

Fig. 6 Principle of the Kolmogorov-Smirnov test.

The upper and lower deviation $d_{U, i}$ and $d_{L, i}$ at $x_{i}$ the are visualized in Fig. 6. The two distributions are accepted to coincide if

$$
d_{\max } \leq d_{\text {allow }}(m, \alpha)
$$

where the allowed value $d_{\text {allow }}$ depends on the number of samples $m$ and the level of significance $\alpha$. The function $d_{\text {allow }}(m, \alpha)$ is givenby [2]

$$
d_{\text {allow }}(m, \alpha=5 \%)=\frac{1.36}{\sqrt{m}} \text { for } m>50
$$

For checking the convergence behavior for an increasing number of plies, two layup types are considered. One layup is chosen to be symmetrical and only consists of $0^{\circ}, 90^{\circ}, 45^{\circ}$ and $-45^{\circ}$ plies. The other layup is generated randomly, where each ply orientation takes values between $0^{\circ}$ and $90^{\circ}$ (see Table 1 ). For both layup types, the ply orientation of each layer is assumed to scatter uniformly at intervals of $\pm 8.66^{\circ}$ around the nominal value. For each ply number $n, 20$ different random laminates are generated and 20 Monte Carlo simulations are performed. For the convergence study shown in Fig. 7, the maximum $d_{\max }$ of all lamination parameters and all Monte Carlo simulations 
is considered. The convergence towards normal distribution, indicated by decreasing values of $d_{\max }$, looks similar for different numbers of samples $m$ (compare Fig. 7, left and right). However, it is difficult to say for which number of plies $n$ it is valid assume normal distribution, because the allowed value $d_{\text {allow }}$ decreases for an increasing number of samples $m$, as given by (40).

Table 1: Examples of laminates used for the convergence study

\begin{tabular}{ccc}
\hline Number of plies $n$ & $\left(0^{\circ}, 90^{\circ}, \pm 45^{\circ}\right)$ layup & $\begin{array}{c}\text { Random layup } \\
{\left[r_{1}, r_{2}, \ldots, r_{n}\right] \text { with } 0^{\circ} \leq r_{i} \leq 90^{\circ}}\end{array}$ \\
\hline 2 & {$[0,0]$} & {$[10.5,77.6]$} \\
3 & {$[0,90,0]$} & {$[21.1,70.1,13.1]$} \\
4 & {$[0,90,90,0]$} & {$[75.8,86.2,49.6,12.4]$} \\
5 & {$[0,90,45,90,0]$} & {$[67.4,4.5,38.6,30.4,55.9]$} \\
$\vdots$ & $\vdots$ & $\vdots$ \\
\hline \hline
\end{tabular}
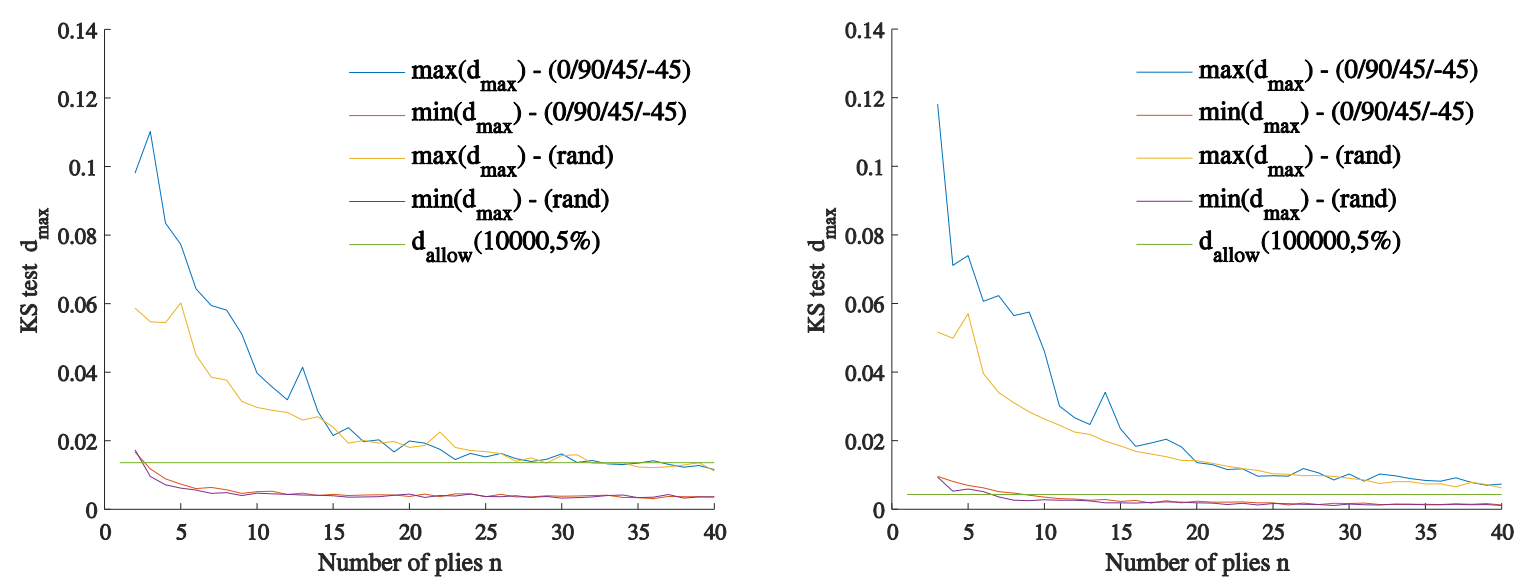

Fig. 7 Results of KS tests for increasing number of plies, Monte Carlo simulations with 10000 samples (left) and with 100000 samples (right). The maximum $d_{\max }$ of all lamination parameters is considered.

If the lamination parameters are normally distributed, the dependence is linear and the Pearson correlation coefficient $\rho_{P}$ and the Spearman's rank correlation coefficient $\rho_{S}$ should have the same value. The Pearson correlation coefficient of two parameters $X$ and $Y$ equals the covariance divided by the product of the standard deviations [2]. 


$$
\rho_{P}=\frac{\operatorname{cov}(X, Y)}{\sigma_{X} \sigma_{Y}}
$$

For the Spearman's rank correlation coefficient, the Pearson correlation coefficient is determined for the ranks $r g$ of the parameters $X$ and $Y$ [24].

$$
\rho_{S}=\frac{\operatorname{cov}\left(r g_{X}, r g_{Y}\right)}{\sigma_{r g_{X}} \sigma_{r g_{Y}}}
$$

Two examples for the evolution of both coefficients for an increasing number of plies is given in Fig. 8. For these examples, the random layup is used. For the convergence check of the correlation, the deviation of Pearson and Spearman's rank correlation $\Delta_{\rho}=\rho_{P}-\rho_{S}$ is considered. In order to get an overview of the correlation of all lamination parameters, the absolute maximum deviation $\max \left(\left|\Delta_{\rho}\right|\right)$ is considered. For the convergence study shown in Fig. 9, 20 different random laminates are generated for each $n$ and the maximum deviation of all combinations of lamination parameters and all Monte Carlo simulations per ply number is taken. The Pearson and Spearman's rank correlation coefficients are already very similar for a small number of plies. Hence, there is no nonlinear dependence which contradicts the assumption of normal distribution.

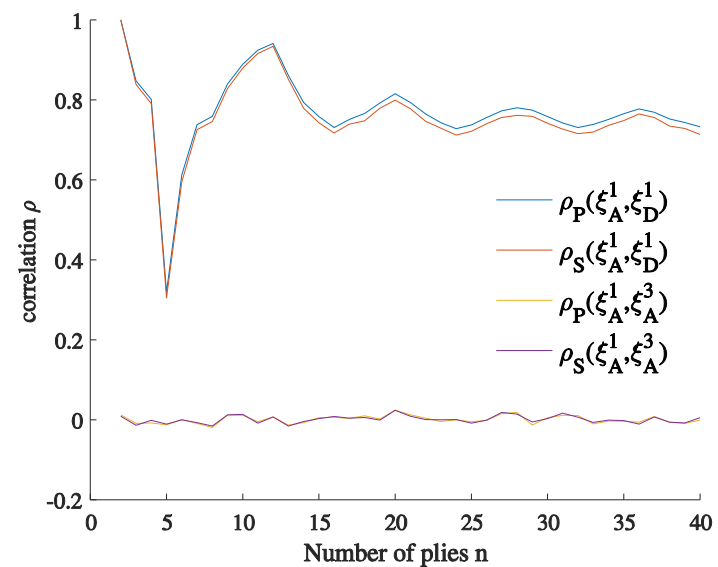

Fig. 8 Comparison of Pearson and Spearman's rank correlation coefficients for selected lamination parameters for an increasing number of plies

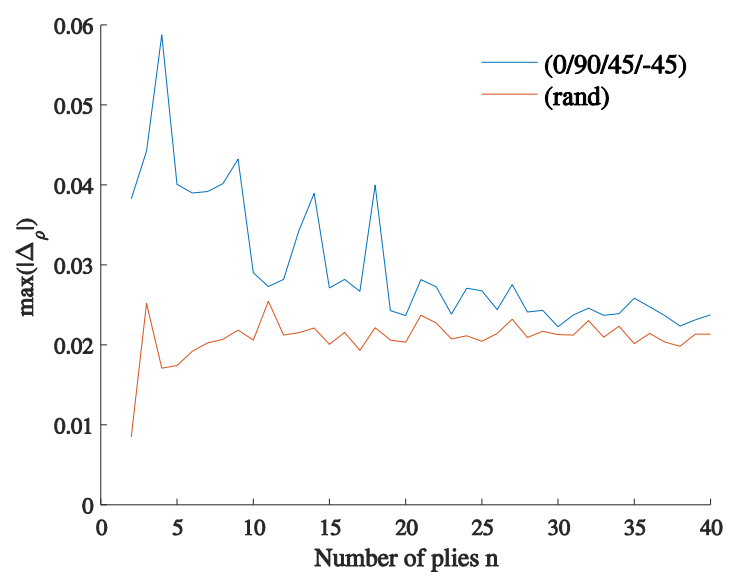

Fig. 9 Maximal difference of Pearson and Spearman's rank correlation coefficients for an increasing number of plies

If the lamination parameters are normally distributed, their skewnesses equals zero. A skewness of zero is no proof of normal distribution, since there are a lot of other distribution types with a skewness of zero. This criterion is therefore considered as necessary, but not sufficient. The convergence of the maximum and minimum skewness of all 
lamination parameters is given in Fig. 10. The study has been performed in the same manner as for the KS test and the correlation coefficients The skewness converges to values between -0.2 and 0.1 . For comparison, the skewness of the Gumbel distribution equals 1.14 .

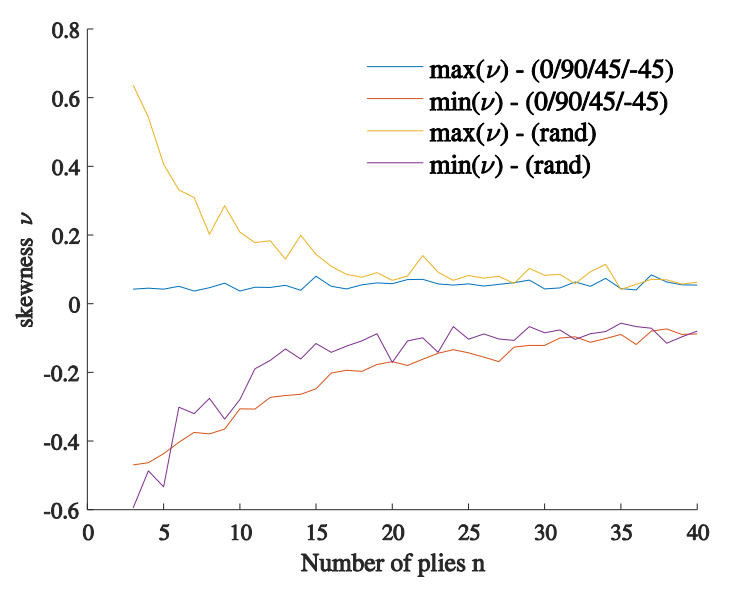

\section{Fig. 10 Skewness for an increasing number of plies}

The studies presented above are performed with two types of laminates (random and $0^{\circ}, 90^{\circ}, \pm 45^{\circ}$ ). The ply angles are assumed to be uniformly distributed in an interval of $\pm 8.66^{\circ}$ around the nominal value. All plies are assumed to have the same thickness and the same distribution. Further studies are performed to investigate the influence of these assumptions, which are summarized briefly in the following. Changing the type of distribution or increasing the standard deviation of the ply orientations has practically no influence on the convergence towards normal distribution. In addition, considering a highly oriented layup (e.g., only $0^{\circ}$ plies) has little influence on the convergence. If the ply thicknesses and/or standard deviation is not the same in all plies, this can have a significant influence on the convergence. For instance, doubling the standard deviation of every $4^{\text {th }}$ ply in a $\left(0^{\circ}, 90^{\circ}, \pm 45^{\circ}\right)$ type laminate doubles the number of plies for which convergence is observed.

\section{Consequences of assuming normal distribution}

If lamination parameters are normally distributed, it is sufficient to determine their mean vector and covariance matrix as described in section III for obtaining the joint distribution function. This distribution is a required input for the actual probabilistic analysis of a structural response (step 2 in Fig. 2).

If this second step is performed using the FOSM method, only the mean vector and covariance matrix of the lamination parameter are required. However, if the structural response is approximately linear (which is implicitly 
assumed by FOSM), the assumption of normally distributed lamination parameters allows assuming a normal distribution for the structural response as well.

Concerning the application of FORM, the transformation to uncorrelated normalized parameters is simply the linear transformation given by (4), if the lamination parameters are normally distributed.

The previous section indicates that if a laminate consists of plies with similar thicknesses and similar scatter of the ply angles, the lamination parameters are approximately normally distributed for a large number of plies. Based on the studies carried out, the author believes that for laminates with more than 20 plies it is reasonable to work with Gaussian distribution. For some lamination parameters the maximum deviations from normal distribution $d_{\text {max }}$ still exceed the allowable value of the K-S test for 20 plies, but this allowable depends on the number of samples considered and furthermore, all other measures converge for 20 plies. However, it needs to be emphasized that lamination parameters are never really normally distributed, because no laminate has an infinite number of plies, and, moreover, lamination parameters are bounded (see, e.g., [25]). In the framework of a Monte Carlo simulation or MPP search, the bounds can be accounted for by using the truncated normal distribution given in [3].

\section{V.Conclusion}

The current paper suggests an efficient methodology for considering ply orientations of composite structures as random parameters within a probabilistic analysis by utilizing lamination parameters. In the first step, the joint stochastic distribution of lamination parameters is determined and, in the second step, the actual probabilistic analysis is performed considering lamination parameters as random parameters. The methodology is relevant for laminates with more than 12 plies, because it increases the number of random variables for fewer plies.

Closed form solutions for the mean values, variances and covariances of lamination parameters are provided. Supposing that the lamination parameters are normally distributed, their distribution is completely described by these stochastic moments.

It is discussed that for laminates consisting of many plies with similar thicknesses and scatter in a ply orientation, the distribution of lamination parameters is approximately Gaussian. Based on convergence studies considering different random and typical laminates, the author believes that for laminates with more than 20 plies it is reasonable

to assume normal distribution. It is emphasized, however, that normal distribution is never the real distribution of lamination parameters, but can only be an approximation. It seems difficult to generally conclude under which 
circumstances normal distribution is sufficiently accurate. The present work is supposed to support the reader in this case-by-case decision.

One example of an application is a robust design optimization, in which reduced accuracy of the probabilistic analysis is acceptable during the optimization. Another possible application is the analysis of thin ply composites, which typically consist of a large number of similar plies.

\section{Appendix}

\section{A. Lamination parameters}

Lamination parameters have been introduced by Tsai and Hahn [8], where in [8] lamination parameters are referred to as "geometric factors". The notation used in the following, however, is taken from [14].

The lamination parameters are defined as

$$
\begin{aligned}
& \xi_{[1,2,3,4]}^{A}=\frac{1}{h} \int_{-h / 2}^{h / 2}[\cos (2 \varphi), \cos (4 \varphi), \sin (2 \varphi), \sin (4 \varphi)] d z \\
& \xi_{[1,2,3,4]}^{B}=\frac{4}{h^{2}} \int_{-h / 2}^{h / 2}[\cos (2 \varphi), \cos (4 \varphi), \sin (2 \varphi), \sin (4 \varphi)] z d z \\
& \xi_{[1,2,3,4]}^{D}=\frac{12}{h^{3}} \int_{-h / 2}^{h / 2}[\cos (2 \varphi), \cos (4 \varphi), \sin (2 \varphi), \sin (4 \varphi)] z^{2} d z
\end{aligned}
$$

where $\varphi$ is the material orientation, $h$ is the whole laminate thickness and $z$ is the through thickness coordinate. When considering a discrete layup, the ply orientations $\varphi_{i}$ and thereby the terms in parentheses are piecewise constant with respect to $z$. The lamination parameters are then computed by

$$
\begin{aligned}
& \xi_{[1,2,3,4]}^{A}=\frac{1}{h} \sum_{i=1}^{n}\left[\cos \left(2 \varphi_{i}\right), \cos \left(4 \varphi_{i}\right), \sin \left(2 \varphi_{i}\right), \sin \left(4 \varphi_{i}\right)\right] t_{p, i} \\
& \xi_{[1,2,3,4]}^{B}=\frac{4}{h^{2}} \sum_{i=1}^{n}\left[\cos \left(2 \varphi_{i}\right), \cos \left(4 \varphi_{i}\right), \sin \left(2 \varphi_{i}\right), \sin \left(4 \varphi_{i}\right)\right] z_{i} t_{p, i} \\
& \xi_{[1,2,3,4]}^{D}=\frac{12}{h^{3}} \sum_{i=1}^{n}\left[\cos \left(2 \varphi_{i}\right), \cos \left(4 \varphi_{i}\right), \sin \left(2 \varphi_{i}\right), \sin \left(4 \varphi_{i}\right)\right]\left(z_{i}^{2} t_{p, i}+\frac{t_{p, i}^{3}}{12}\right)
\end{aligned}
$$

where $\varphi_{i}$ is the ply orientation, $t_{p, i}$ the thickness and $z_{i}$ the center of gravity of the $i$-th ply. 
Given the lamination parameter, the $\mathrm{ABD}$ matrix is computed by

$$
\begin{aligned}
& \left(\begin{array}{l}
A_{11} \\
A_{22} \\
A_{12} \\
A_{33} \\
A_{13} \\
A_{23}
\end{array}\right)=h\left(\begin{array}{ccccc}
1 & \xi_{1}^{A} & \xi_{2}^{A} & 0 & 0 \\
1 & -\xi_{1}^{A} & \xi_{2}^{A} & 0 & 0 \\
0 & 0 & -\xi_{2}^{A} & 1 & 0 \\
0 & 0 & -\xi_{2}^{A} & 0 & 1 \\
0 & \frac{1}{2} \xi_{3}^{A} & \xi_{4}^{A} & 0 & 0 \\
0 & \frac{1}{2} \xi_{3}^{A} & -\xi_{4}^{A} & 0 & 0
\end{array}\right)\left(\begin{array}{l}
U_{1} \\
U_{2} \\
U_{3} \\
U_{4} \\
U_{5}
\end{array}\right) \\
& \left(\begin{array}{l}
B_{11} \\
B_{22} \\
B_{12} \\
B_{33} \\
B_{13} \\
B_{23}
\end{array}\right)=\frac{h^{2}}{4}\left(\begin{array}{ccccc}
0 & \xi_{1}^{B} & \xi_{2}^{B} & 0 & 0 \\
0 & -\xi_{1}^{B} & \xi_{2}^{B} & 0 & 0 \\
0 & 0 & -\xi_{2}^{B} & 0 & 0 \\
0 & 0 & -\xi_{2}^{B} & 0 & 0 \\
0 & \frac{1}{2} \xi_{3}^{B} & \xi_{4}^{B} & 0 & 0 \\
0 & \frac{1}{2} \xi_{3}^{B} & -\xi_{4}^{B} & 0 & 0
\end{array}\right)\left(\begin{array}{c}
U_{1} \\
U_{2} \\
U_{3} \\
U_{4} \\
U_{5}
\end{array}\right) \\
& \left(\begin{array}{l}
D_{11} \\
D_{22} \\
D_{12} \\
D_{33} \\
D_{13} \\
D_{23}
\end{array}\right)=\frac{h^{3}}{12}\left(\begin{array}{ccccc}
1 & \xi_{1}^{D} & \xi_{2}^{D} & 0 & 0 \\
1 & -\xi_{1}^{D} & \xi_{2}^{D} & 0 & 0 \\
0 & 0 & -\xi_{2}^{D} & 1 & 0 \\
0 & 0 & -\xi_{2}^{D} & 0 & 1 \\
0 & \frac{1}{2} \xi_{3}^{D} & \xi_{4}^{D} & 0 & 0 \\
0 & \frac{1}{2} \xi_{3}^{D} & -\xi_{4}^{D} & 0 & 0
\end{array}\right)\left(\begin{array}{l}
U_{1} \\
U_{2} \\
U_{3} \\
U_{4} \\
U_{5}
\end{array}\right)
\end{aligned}
$$

where the vector of invariants $\mathbf{U}$ is given by

$$
\left(\begin{array}{l}
U_{1} \\
U_{2} \\
U_{3} \\
U_{4} \\
U_{5}
\end{array}\right)=\frac{1}{8}\left[\begin{array}{cccc}
3 & 3 & 2 & 4 \\
4 & -4 & 0 & 0 \\
1 & 1 & -2 & -4 \\
1 & 1 & 6 & -4 \\
1 & 1 & -2 & 4
\end{array}\right]\left(\begin{array}{c}
Q_{11} \\
Q_{22} \\
Q_{12} \\
Q_{66}
\end{array}\right)
$$

with the in plane stiffness properties

$$
\begin{aligned}
& Q_{11}=\frac{E_{11}}{1-v_{12} v_{21}} \quad Q_{22}=\frac{E_{22}}{1-v_{12} v_{21}} \\
& Q_{12}=v_{12} Q_{22} \quad Q_{66}=G_{12} \quad v_{21}=v_{12} \frac{E_{22}}{E_{11}}
\end{aligned}
$$


The material parameters in (49) are the Young's modulus in fiber direction $E_{11}$, the Young's modulus perpendicular to fiber direction $E_{22}$, the shear modulus $G_{12}$ and the Poisson's ratio $v_{12}$. The material properties used in the present paper are taken from [21].

\section{B. Mean value of sine and cosine}

In section III, the stochastic moments (mean value, variance and covariance) of the lamination parameters are derived, which require the stochastic moments of sine and cosine. These are derived in the following.

Introduce

$$
c_{a}=\cos (a x), s_{b}=\sin (b x)
$$

For any function $g(x)$ depending on a random parameter $x$, the mean value is given by

$$
E(g)=\int_{-\infty}^{\infty} g(x) f_{g}(g) d g=\int_{-\infty}^{\infty} g(x) f_{X}(x) d x
$$

Hence, for sine and cosine the mean values are

$$
E\left(c_{a}\right)=\int_{-1}^{1} c_{a} f_{C_{a}}\left(c_{a}\right) d c_{a}=\int_{-\infty}^{\infty} \cos (a x) f_{X}(x) d x
$$

and

$$
E\left(s_{b}\right)=\int_{-1}^{1} s_{b} f_{S_{b}}\left(s_{b}\right) d s_{b}=\int_{-\infty}^{\infty} \sin (b x) f_{X}(x) d x
$$

For certain cases of $f_{X}(x)$, the integrals (52) and (53) can be solved directly, as given in Appendix D. In any case, cosine and sine can be approximated by Taylor series expansions.

$$
\begin{gathered}
\cos (a x)=\sum_{j=0}^{\infty}(-1)^{j} \frac{(a x)^{2 j}}{(2 j) !}=\sum_{j=0}^{\infty} \frac{(-1)^{j}}{(2 j) !} a^{2 j} x^{2 j} \\
\sin (b x)=\sum_{j=0}^{\infty}(-1)^{j} \frac{(b x)^{2 j+1}}{(2 j+1) !}=\sum_{j=0}^{\infty} \frac{(-1)^{j}}{(2 j+1) !} b^{2 j+1} x^{2 j+1}
\end{gathered}
$$

Inserting the Taylor series into (52) and (53) yields 


$$
\begin{aligned}
E\left(c_{a}\right) & =\int_{-\infty}^{\infty}\left[\sum_{j=0}^{\infty} \frac{(-1)^{j}}{(2 j) !} a^{2 j} x^{2 j}\right] f_{X}(x) d x=\sum_{j=0}^{\infty} \frac{(-1)^{j}}{(2 j) !} a^{2 j} \underbrace{\int_{-\infty}^{\infty} x^{2 j} f_{X}(x) d x}_{E\left(X^{2 j}\right)} \\
& =\sum_{j=0}^{\infty} \frac{(-1)^{j}}{(2 j) !} a^{2 j} E\left(X^{2 j}\right)
\end{aligned}
$$

and

$$
\begin{aligned}
E\left(s_{b}\right) & =\int_{-\infty}^{\infty}\left[\sum_{j=0}^{\infty} \frac{(-1)^{j}}{(2 j+1) !} b^{2 j+1} x^{2 j+1}\right] f_{X}(x) d x=\sum_{j=0}^{\infty} \frac{(-1)^{j}}{(2 j+1) !} b^{2 j+1} \underbrace{\int_{-\infty}^{\infty} x^{2 j+1} f_{X}(x) d x}_{E\left(X^{2 j+1}\right)} \\
& =\sum_{j=0}^{\infty} \frac{(-1)^{j}}{(2 j+1) !} b^{2 j+1} E\left(X^{2 j+1}\right)
\end{aligned}
$$

Equations (56) and (57) require determining the stochastic moments $E\left(X^{k}\right)$, which can be determined from the distribution of $X$. For any type of distribution, the $k$-th stochastic moment is given by the $k$-th derivative of the moment generating function $M_{X}(t)$ with respect to $t$ at $t=0$ [23].

$$
E\left(X^{k}\right)=\frac{d^{k} M_{X}(0)}{d t^{k}}
$$

A list of moment generating functions for different types of distribution is, for instance, given in [26]. For normal distribution, all central moments are given by the equation below [27].

$$
E\left(X^{k}\right)=\sum_{i=0}^{k / 2}\left(\begin{array}{c}
k \\
2 i
\end{array}\right)(2 i-1) ! ! \sigma^{2 i} \mu^{k-2 i}
$$

The series in (56) and (57) must have a finite upper bound and, therefore, the results must be considered as an approximation of the mean value. The convergence of this approximation depends on the ply angle. For normally distributed ply angles with a variance of $8.33^{\circ}$ (derived from a $\pm 5^{\circ}$ interval, see IV.B), the recommendations given in Table 2 are derived from a convergence study.

Table 2: Recommended upper bounds of series for mean value approximations (56) and (57)

\begin{tabular}{cccc}
\hline \hline Ply angle & $0^{\circ}$ & $\pm 45^{\circ}$ & $90^{\circ}$ \\
\hline Upper bound of series & 5 & 13 & 21 \\
\hline \hline
\end{tabular}




\section{Mean of the product of trigonometric functions}

The derivation of covariances of lamination parameters in section III.D requires the joint moments of trigonometric functions for all combinations of $c_{2, i}, c_{4, i}, s_{2, i}$ and $s_{4, i}$. For this, $\chi_{k, i}$ (or $\chi_{l, j}$ respectively) are introduced, which represent any trigonometric function of $k \varphi_{i}$ (or $\left.l \varphi_{j}\right)$; see (27).

The joint moment is the moment of the product of the trigonometric functions

$$
E\left(\chi_{k, i} \chi_{l, j}\right)=\int_{-1}^{1} \int_{-1}^{1} \chi_{k, i} \chi_{l, j} f_{\mathrm{X}_{k, i}, \mathrm{X}_{l, j}}\left(\chi_{k, i}, \chi_{l, j}\right) d \chi_{l, j} d \chi_{k, i}
$$

where $f_{\mathrm{X}_{k, i}, \mathrm{X}_{l, j}}\left(\chi_{k, i}, \chi_{l, j}\right)$ is the joint probability density function (PDF). As $\chi_{k, i}$ and $\chi_{l, j}$ are functions of $k \varphi_{i}$ and $l \varphi_{j}$, making use of (51) yields

$$
E\left(\chi_{k, i} \chi_{l, j}\right)=\int_{-\infty}^{\infty} \int_{-\infty}^{\infty} \chi_{k, i} \chi_{l, j} f_{\Phi_{i}, \Phi_{i}}\left(\varphi_{i}, \varphi_{j}\right) d \varphi_{j} d \varphi_{i}
$$

It is now worth differentiating between the two cases $i=j$ and $i \neq j$. For $i \neq j, \varphi_{i}$ and $\varphi_{j}$ are independent and their joint PDF is the product of the individual PDFs, which yields

$$
\begin{aligned}
E\left(\chi_{k, i} \chi_{l, j}\right) & =\int_{-\infty}^{\infty} \int_{-\infty}^{\infty} \chi_{k, i} \chi_{l, j} f_{\Phi_{i}, \Phi_{i}}\left(\varphi_{i}, \varphi_{j}\right) d \varphi_{j} d \varphi_{i} \\
& =\int_{-\infty}^{\infty} \int_{-\infty}^{\infty} \chi_{k, i} \chi_{l, j} f_{\Phi_{i}}\left(\varphi_{i}\right) f_{\Phi_{j}}\left(\varphi_{j}\right) d \varphi_{j} d \varphi_{i} \quad \text { for } i \neq j \\
& =\int_{-\infty}^{\infty} \chi_{k, i} f_{\Phi_{i}}\left(\varphi_{i}\right) d \varphi_{i} \int_{-\infty}^{\infty} \chi_{l, j} f_{\Phi_{j}}\left(\varphi_{j}\right) d \varphi_{j}=E\left(\chi_{k, i}\right) E\left(\chi_{l, j}\right)
\end{aligned}
$$

Hence, for $i \neq j$ the joint moment equals the product of the individual moments.

For $i=j$, the joint PDF of $\varphi_{i}$ and $\varphi_{j}$ is simply the PDF of $\varphi_{i}$, which yields

$$
E\left(\chi_{k, i} \chi_{l, i}\right)=\int_{-\infty}^{\infty} \chi_{k, i} \chi_{l, i} f_{\Phi_{i}}\left(\varphi_{i}\right) d \varphi_{i}
$$

The integral (63) needs to be solved for the following combinations.

$$
E\left(c_{a, i} c_{b, i}\right)=\int_{-\infty}^{\infty} \cos \left(a \varphi_{i}\right) \cos \left(b \varphi_{i}\right) f_{\Phi_{i}}\left(\varphi_{i}\right) d \varphi_{i}
$$




$$
\begin{aligned}
& E\left(s_{a, i} s_{b, i}\right)=\int_{-\infty}^{\infty} \sin \left(a \varphi_{i}\right) \sin \left(b \varphi_{i}\right) f_{\Phi_{i}}\left(\varphi_{i}\right) d \varphi_{i} \\
& E\left(c_{a, i} s_{b, i}\right)=\int_{-\infty}^{\infty} \cos \left(a \varphi_{i}\right) \sin \left(b \varphi_{i}\right) f_{\Phi_{i}}\left(\varphi_{i}\right) d \varphi_{i}
\end{aligned}
$$

where $a$ and $b$ equal 2 or 4 .

These integrals can either be solved directly when the PDF allows it (see, e.g., Appendix D), or the trigonometric functions are approximated using the Taylor series.

Inserting the series expansion of cosine (54) into (64) yields

$$
\begin{aligned}
E\left(c_{a, i} c_{b, i}\right) & =\int_{-\infty}^{\infty}\left[\sum_{j=0}^{\infty} \frac{(-1)^{j}}{(2 j) !} a^{2 j} \varphi_{i}^{2 j}\right]\left[\sum_{k=0}^{\infty} \frac{(-1)^{k}}{(2 k) !} b^{2 k} \varphi_{i}^{2 k}\right] f_{\Phi_{i}}\left(\varphi_{i}\right) d \varphi_{i} \\
& =\sum_{j=0}^{\infty} \sum_{k=0}^{\infty} \frac{(-1)^{j}}{(2 j) !} a^{2 j} \frac{(-1)^{k}}{(2 k) !} b^{2 k} \underbrace{\int_{-\infty}^{\infty} \varphi_{i}^{2 j+2 k} f_{\Phi_{i}}\left(\varphi_{i}\right) d \varphi_{i}}_{E\left(\varphi_{i}^{2 j+2 k}\right)} \\
& =\sum_{j=0}^{\infty} \sum_{k=0}^{\infty} \frac{(-1)^{j}}{(2 j) !} a^{2 j} \frac{(-1)^{k}}{(2 k) !} b^{2 k} E\left(\varphi_{i}^{2 j+2 k}\right)
\end{aligned}
$$

Inserting the series expansion of sine (55) into (65) yields

$$
\begin{aligned}
E\left(s_{a, i} s_{b, i}\right) & =\int_{-\infty}^{\infty}\left[\sum_{j=0}^{\infty} \frac{(-1)^{j}}{(2 j+1) !} a^{2 j+1} \varphi_{i}^{2 j+1}\right]\left[\sum_{k=0}^{\infty} \frac{(-1)^{j}}{(2 k+1) !} b^{2 k+1} \varphi_{i}^{2 k+1}\right] f_{\Phi_{i}}\left(\varphi_{i}\right) d \varphi_{i} \\
& =\sum_{j=0}^{\infty} \sum_{k=0}^{\infty} \frac{(-1)^{j}}{(2 j+1) !} a^{2 j+1} \frac{(-1)^{j}}{(2 k+1) !} b^{2 k+1} \underbrace{\int_{-\infty}^{\infty} \varphi_{i}^{2 j+2 k+2} f_{\Phi_{i}}\left(\varphi_{i}\right) d \varphi_{i}}_{E\left(\varphi_{i}^{2 j+2 k+2}\right)} \\
& =\sum_{j=0}^{\infty} \sum_{k=0}^{\infty} \frac{(-1)^{j}}{(2 j+1) !} a^{2 j+1} \frac{(-1)^{k}}{(2 k+1) !} b^{2 k+1} E\left(\varphi_{i}^{2 j+2 k+2}\right)
\end{aligned}
$$

Inserting the series expansion of cosine (54) and sine (55) into (66) yields 


$$
\begin{aligned}
E\left(c_{a, i} s_{b, i}\right) & =\int_{-\infty}^{\infty}\left[\sum_{j=0}^{\infty} \frac{(-1)^{j}}{(2 j) !} a^{2 j} \varphi_{i}^{2 j}\right]\left[\sum_{k=0}^{\infty} \frac{(-1)^{j}}{(2 k+1) !} b^{2 k+1} \varphi_{i}^{2 k+1}\right] f_{\Phi_{i}}\left(\varphi_{i}\right) d \varphi_{i} \\
& =\sum_{j=0}^{\infty} \sum_{k=0}^{\infty} \frac{(-1)^{j}}{(2 j) !} a^{2 j} \frac{(-1)^{j}}{(2 k+1) !} b^{2 k+1} \underbrace{\int_{-\infty}^{\infty} \varphi_{i}^{2 j+2 k+1} f_{\Phi_{i}}\left(\varphi_{i}\right) d \varphi_{i}}_{E\left(\varphi_{i}^{2 j+2 k+1}\right)} \\
& =\sum_{j=0}^{\infty} \sum_{k=0}^{\infty} \frac{(-1)^{j}}{(2 j) !} a^{2 j} \frac{(-1)^{j}}{(2 k+1) !} b^{2 k+1} E\left(\varphi_{i}^{2 j+2 k+1}\right)
\end{aligned}
$$

Summarized, the joint moment of two trigonometric functions is given by (62) for $i \neq j$ and otherwise by (67)-(69).

\section{Direct solution of trigonometric function integrals for uniform distribution}

As derived in Appendix B, the stochastic moments are given by

$$
E\left(c_{a}\right)=\int_{-\infty}^{\infty} \cos (a x) f_{X}(x) d x
$$

and

$$
E\left(s_{b}\right)=\int_{-\infty}^{\infty} \sin (b x) f_{X}(x) d x
$$

In this section, consider uniform distribution with the PDF

$$
f_{X}(x)= \begin{cases}\frac{1}{d-c} & \text { for } c \leq x \leq d \\ 0 & \text { else }\end{cases}
$$

Inserting (72) into (70) yields

$$
\begin{aligned}
& E\left(c_{a}\right)=\int_{c}^{d} \cos (a x) \frac{1}{d-c} d x=\frac{1}{d-c} \int_{c}^{d} \cos (a x) d x \\
& =\frac{1}{d-c}\left[\frac{1}{a} \sin (a x)\right]_{c}^{d}=\frac{1}{d-c} \frac{1}{a}[\sin (a d)-\sin (a c)]
\end{aligned}
$$

and inserting (72) into (53) yields 


$$
\begin{aligned}
& E\left(s_{b}\right)=\int_{c}^{d} \sin (b x) \frac{1}{d-c} d x=\frac{1}{d-c} \int_{c}^{d} \sin (b x) d x \\
& =\frac{1}{d-c}\left[-\frac{1}{b} \cos (b x)\right]_{c}^{d}=-\frac{1}{d-c} \frac{1}{b}[\cos (b d)-\cos (b c)]
\end{aligned}
$$

For the determination of covariances in section III.D, the joint moments $E\left(c_{a} c_{b}\right), E\left(s_{a} s_{b}\right)$ and $E\left(c_{a} s_{b}\right)$ need to be determined, which is done for uniform distribution in the following manner.

$$
E\left(c_{a} c_{b}\right)=\int_{-\infty}^{\infty} \cos (a x) \cos (b x) f_{X}(x) d x=\frac{1}{d-c} \int_{c}^{d} \cos (a x) \cos (b x) d x
$$

The case $a \neq b$ yields

$$
\begin{aligned}
E\left(c_{a} c_{b}\right) & =\frac{1}{d-c}\left[\frac{\sin (a-b) x}{2(a-b)}+\frac{\sin (a+b) x}{2(a+b)}\right]_{c}^{d} \\
& =\frac{1}{d-c}\left[\frac{\sin (a-b) d}{2(a-b)}+\frac{\sin (a+b) d}{2(a+b)}-\frac{\sin (a-b) c}{2(a-b)}-\frac{\sin (a+b) c}{2(a+b)}\right]
\end{aligned}
$$

where for $a=b$

$$
\begin{aligned}
E\left(c_{a}^{2}\right) & =\frac{1}{d-c} \int_{c}^{d} \cos ^{2}(a x) d x=\frac{1}{d-c}\left[\frac{x}{2}+\frac{1}{4 a} \sin (2 a x)\right]_{c}^{d} \\
& =\frac{1}{d-c}\left[\frac{d}{2}+\frac{1}{4 a} \sin (2 a d)-\frac{c}{2}-\frac{1}{4 a} \sin (2 a c)\right]
\end{aligned}
$$

The joint moment of $E\left(s_{a} s_{b}\right)$ equals

$$
E\left(s_{a} s_{b}\right)=\int_{-\infty}^{\infty} \sin (a x) \sin (b x) f_{X}(x) d x=\frac{1}{d-c} \int_{c}^{d} \sin (a x) \sin (b x) d x
$$

For $a \neq b$

$$
\begin{aligned}
E\left(s_{a} s_{b}\right) & =\frac{1}{d-c}\left[\frac{\sin (a-b) x}{2(a-b)}-\frac{\sin (a+b) x}{2(a+b)}\right]_{c}^{d} \\
& =\frac{1}{d-c}\left[\frac{\sin (a-b) d}{2(a-b)}-\frac{\sin (a+b) d}{2(a+b)}-\frac{\sin (a-b) c}{2(a-b)}+\frac{\sin (a+b) c}{2(a+b)}\right]
\end{aligned}
$$

and for $a=b$ 


$$
\begin{aligned}
& E\left(s_{a}^{2}\right)=\frac{1}{d-c} \int_{c}^{d} \sin ^{2}(a x) d x=\frac{1}{d-c}\left[\frac{x}{2}-\frac{1}{4 a} \sin (2 a x)\right]_{c}^{d} \\
& =\frac{1}{d-c}\left[\frac{d}{2}-\frac{1}{4 a} \sin (2 a d)-\frac{c}{2}+\frac{1}{4 a} \sin (2 a c)\right]
\end{aligned}
$$

Finally, the joint moment of $E\left(s_{a} s_{b}\right)$ is given by

$$
E\left(c_{a} s_{b}\right)=\int_{-\infty}^{\infty} \sin (a x) \cos (b x) f_{X}(x) d x=\frac{1}{d-c} \int_{-\infty}^{\infty} \sin (a x) \cos (b x) d x
$$

For $a \neq b$

$$
\begin{aligned}
E\left(c_{a} s_{b}\right) & =\frac{1}{d-c}\left[-\frac{\cos (a+b) x}{2(a+b)}-\frac{\cos (b-a) x}{2(b-a)}\right]_{c}^{d} \\
& =\frac{1}{d-c}\left[-\frac{\cos (a+b) d}{2(a+b)}-\frac{\cos (b-a) d}{2(b-a)}+\frac{\cos (a+b) c}{2(a+b)}+\frac{\cos (b-a) c}{2(b-a)}\right]
\end{aligned}
$$

and for $a=b$

$$
\begin{aligned}
E\left(c_{a} s_{a}\right) & =\int_{-\infty}^{\infty} \sin (a x) \cos (a x) f_{X}(x) d x=\frac{1}{d-c} \int_{c}^{d} \sin (a x) \cos (a x) d x \\
& =\frac{1}{d-c}\left[\frac{\sin ^{2}(a x)}{2 a}\right]_{c}^{d}=\frac{1}{d-c}\left[\frac{\sin ^{2}(a d)}{2 a}-\frac{\sin ^{2}(a c)}{2 a}\right]
\end{aligned}
$$

In a similar way, the moments can be determined analytically for the Simpson distribution. For normal distribution, however, a closed form solution of the integral is not possible.

\section{Acknowledgments}

The support of Dr. J. Enrique Herencia in preparing this manuscript is gratefully acknowledged. Without his support, especially on the concept of lamination parameters, this paper could not have been written.

\section{References}

[1] M. W. Long and J. D. Narciso, "Probabilistic Design Methodology for Composite Aircraft Structures.," 1999, DTIC Document.

[2] A. Haldar and S. Mahadevan, Probability, Reliability, and Statistical Methods in Engineering Design. John Wiley \& Sons New York/Chichester, UK, 2000.

[3] I. Elishakof, Probabilistic Theory of Structures. Mineola, NY: Dover, 1999. 
[4] R. Degenhardt, A. Kling, A. Bethge, J. Orf, L. Kärger, R. Zimmermann, K. Rohwer, and A. Calvi, "Investigations on Imperfection Sensitivity and Deduction of Improved Knock-down Factors for Unstiffened CFRP Cylindrical Shells," Composite Structures, 92(8), 1939-1946, 2010.

[5] B. Kriegesmann, E. L. Jansen, and R. Rolfes, "Semi-analytic probabilistic analysis of axially compressed stiffened composite panels," Composite Structures, 94(2), 654-663, Jan. 2012.

[6] I. Elishakoff, S. van Manen, P. G. Vermeulen, and J. Arbocz, "First-Order Second-Moment Analysis of the Buckling of Shells with Random Imperfections," AIAA journal, 25(8), 1113-1117, 1987.

[7] B. Kriegesmann, R. Rolfes, C. Hühne, and A. Kling, "Fast Probabilistic Design Procedure for Axially Compressed Composite Cylinders," Composites Structures, 93, 3140-3149, 2011.

[8] S. W. Tsai and H. T. Hahn, Introduction to Composite Materials. CRC Press, 1980.

[9] M. Miki, "Material design of composite laminates with required in-plane elastic properties," in Proceedings of the 4th International Conference on Composite Materials, Tokyo, Japan, 1982, 1725-1731.

[10] H. Fukunaga and Y. Hirano, "Stability optimization of laminated composite plates under in-plane loads," in Proceedings of the 4th International Conference on Composite Materials, Tokyo, Japan, 1982, 565-572.

[11] M. Miki and Y. Sugiyama, "Optimum Design of Laminated Composite Plates Using Lamination Parameters," AIAA Journal, 31(5), 921-922, 1993.

[12] H. Fukunaga and H. Sekine, "Stiffness design method of symmetric laminates using lamination parameters," Aiaa Journal, 30(11), 2791-2793, 1992.

[13] S. Setoodeh, M. M. Abdalla, and Z. Gürdal, "Design of variable-stiffness laminates using lamination parameters,” Composites Part B: Engineering, 37(4-5), 301-309, Jun. 2006.

[14] J. E. Herencia, P. M. Weaver, and M. I. Friswell, "Optimization of long anisotropic laminated fiber composite panels with T-shaped stiffeners," AIAA journal, 45(10), 2497-2509, 2007.

[15] A. Jibawy, C. Julien, B. Desmorat, A. Vincenti, and F. Léné, "Hierarchical structural optimization of laminated plates using polar representation," International Journal of Solids and Structures, 48(18), 2576-2584, Sep. 2011.

[16] M. Montemurro, A. Vincenti, and P. Vannucci, "A Two-Level Procedure for the Global Optimum Design of Composite Modular Structures-Application to the Design of an Aircraft Wing Part 1," Journal of Optimization Theory and Applications, 155(1), 1-23, May 2012.

[17] M. Montemurro, A. Vincenti, and P. Vannucci, "A Two-Level Procedure for the Global Optimum Design of Composite Modular Structures-Application to the Design of an Aircraft Wing Part 2," Journal of Optimization Theory and Applications, 155(1), 24-53, May 2012.

[18] M. Miki, Y. Murotsu, N. Murayama, and T. Tanaka, "Application of Lamination Parameters to ReliabilityBased Stiffness Design of Composites," AIAA journal, 31(10), 1938-1945, 1993.

[19] N. Kogiso, S. Nakagawa, and Y. Murotsu, "Lamination Parameters Applied to Reliability-Based In-Plane Strength Design of Composites," AIAA Journal, 41(11), 2200-2207, 2003.

[20] N. Kogiso, S. Nakagawa, and Y. Murotsu, "Lamination Parameters Applied to Reliability-Based Optimization of Composite Plate," in 9th AIAA/ISSMO Symposium on Multidisciplinary Analysis and Optimization, American Institute of Aeronautics and Astronautics.

[21] C. Scarth, J. E. Cooper, P. M. Weaver, and G. H. C. Silva, "Uncertainty quantification of aeroelastic stability of composite plate wings using lamination parameters," Composite Structures, 116, 84-93, Sep. 2014.

[22] M. Loève, Probability theory I, 4. ed. Springer, 1977.

[23] P. Billingsley, Probability and measure, 3. ed. Wiley, 2012.

[24] W. W. 1929- Daniel, Applied nonparametric statistics, 2. ed. PWS-KENT, 1990.

[25] M. W. Bloomfield, C. G. Diaconu, and P. M. Weaver, "On feasible regions of lamination parameters for layup optimization of laminated composites," Proceedings of the Royal Society of London A: Mathematical, Physical and Engineering Sciences, 465(2104), 1123-1143, Apr. 2009.

[26] "Moment-generating function," Wikipedia, the free encyclopedia, 26-Aug-2015. [Online]. Available: https://en.wikipedia.org/w/index.php?title=Moment-generating_function\&oldid=677874463. [Accessed: 20Sep-2015].

[27] J. D. Cook, "Formula for normal moments." [Online]. Available: http://www.johndcook.com/blog/2012/11/06/general-formula-for-normal-moments/. [Accessed: 20-Sep2015]. 